\title{
Reynolds stress estimation up to single-pixel resolution using PIV-measurements
}

\author{
Sven Scharnowski • Rainer Hain • Christian J. Kähler
}

Received: 1 October 2010/Revised: 23 May 2011/Accepted: 3 August 2011/Published online: 21 August 2011

(C) The Author(s) 2011. This article is published with open access at Springerlink.com

\begin{abstract}
This article derives a theory for estimating Reynolds normal and shear stresses from PIV images with single-pixel resolution. The main idea is the analysis of the correlation function to identify the probability density function from which the Reynolds stresses can be derived in a 2-D regime. The work establishes a theoretical framework including the influence of the particle image diameter and the velocity gradients on the shape of the correlation function. Synthetic data sets are used for the validation of the proposed method. The application of the evaluation method on two experimental data sets shows that high resolution and accuracy are also obtained with experimental data. The approach is very general and can also be applied to correlation peaks that are obtained from sum-of-correlation PIV evaluations.
\end{abstract}

\section{Introduction}

The analysis of PIV recordings with single-pixel resolution or two-point ensemble correlation has been developed during the last years in order to enhance the spatial resolution and to increase the measurement accuracy of small scale flow phenomena (Westerweel et al. 2004). In microfluids, this approach was first applied under the assumption of stationary flows. Later, it was extended for the analysis of periodic (Billy et al. 2004) and fully turbulent flows (Kähler et al. 2006), and the extension to stereoscopic recordings was examined (Scholz and Kähler 2006). The boundary layer measurements in Kähler et al. (2006), as

S. Scharnowski $(\bowtie) \cdot$ R. Hain · C. J. Kähler

Institute of Fluid Mechanics and Aerodynamics, Universität der

Bundeswehr München, 85577 Neubiberg, Germany

e-mail: sven.scharnowski@unibw.de well as those performed by Bitter et al. (2009) using longrange micro-PIV for the flow along a generic space launcher model at supersonic Mach numbers, showed the potential of the single-pixel approach. As this measurement technique is increasingly applied in the turbulent flow regime to resolve near-wall regions or thin shear layers in separated flows, the following question arises: Can the Reynolds normal and shear stresses be computed with single-pixel resolution?

With conventional PIV evaluations, the stresses are directly computed from an ensemble of velocity fields after subtracting the mean field from each sample. These vector fields are obtained from the spatial cross-correlation of interrogation windows of each (single exposed) PIV image pair. The location of the cross-correlation function's maximum corresponds to the mean displacement of the particle images in the interrogation window. Thus, the resolution of the Reynolds stresses is limited by the size of the windows used for the cross-correlation. This means that the computed values only represent an average over the corresponding interrogation area and that strong spatial changes, as the ones that occur in boundary and shear layers or separated regions, are smeared out. Hence, the finite interrogation window size acts as a low-pass filter.

Reducing the window size to enhance the spatial resolution leads to higher measurement noise and thus to an artificial amplification of Reynolds stresses. On top of that, the number of spurious vectors increases (Raffel et al. 2007). A good compromise between accuracy and resolution would be a window size between $16 \mathrm{px} \times 16 \mathrm{px}$ and $32 \mathrm{px} \times 32 \mathrm{px}$, depending on the particle image's size and density.

The two-point ensemble correlation can be used to improve the spatial resolution without lowering the measurement precision, but does not allow for computing an ensemble of independent vector fields. Thus, statistical 
information about the flow cannot be extracted in the same way as in standard PIV methods.

However, statistical variables can be extracted from the shape of the correlation peak. Kähler and Scholz (2006) suggested a method for estimating (symmetric) normally distributed turbulence from the size of the correlation peak. A fully optical approach was performed by Arnold et al. (1986) by analyzing the visibility in speckle velocimetry. Both examples indicate that the probability density function of the velocity can be used to extract the statistical information about the variance of the flow.

This paper will show how to quantitatively estimate the Reynolds normal and shear stresses with up to single-pixel resolution. The article is divided into three main sections. Section 2 discusses the relationship between the probability density function and the correlation function in an analytical way. An analytical equation for the correlation function is derived in order to determine the influence of velocity gradients, the particle image diameter, and the shape of the probability density function on the correlation function. This is followed by a systematic investigation of synthetic PIV recordings in Sect. 3, where the accuracy and the applicability of the new method were studied. Finally, the developed approach is tested on two experimental examples to show the benefit of the increased spatial resolution.

\section{Mathematical description}

This work suggests a method for computing in-plane components of the Reynolds stress tensor from the correlation function. Since the correlation function can be computed for each single-pixel of the PIV images using the two-point ensemble correlation, the suggested method allows for the estimation of the Reynolds stresses with single-pixel resolution. For a sufficient ensemble of PIV image pairs, the shape of a correlation peak is similar to the convolution of the particle image and the probability density function of the velocity even though it is broadened due to the correlation procedure. Without stresses, this peak is narrow and has a distinct maximum. In the case of Reynolds normal stresses, the correlation peaks become broader and shear stresses lead to an asymmetric shape. The connection between the shape of the correlation peak and the existing Reynolds stresses is discussed in this section.

The two-dimensional velocity field is described as follows:

$$
\begin{aligned}
\mathbf{v}(x, y, t) & =\overline{\mathbf{v}}(x, y)+\mathbf{v}^{\prime}(x, y, t) \\
& =\left(\begin{array}{c}
\bar{u}(x, y)+u^{\prime}(x, y, t) \\
\bar{v}(x, y)+v^{\prime}(x, y, t)
\end{array}\right),
\end{aligned}
$$

where $\overline{\mathbf{v}}$ and $\mathbf{v}^{\prime}$ are the mean velocity and the corresponding velocity fluctuation, respectively. The mean velocity can be estimated with single-pixel resolution. However, there is no direct access to the temporal development of the velocity fluctuations but statistical values of the fluctuations can be computed from their probability density function.

The joint probability density function $\operatorname{PDF}(u, v, x, y)$ among the two velocity components includes all occurring velocities for a given point $(x, y)$ over a certain time (or for an ensemble of measurements) and allocates the related probability. Hence, it is possible to compute the Reynolds normal and shear stresses using the probability density function of the velocity field given by:

$$
\begin{aligned}
& \left\langle u^{\prime}(x, y)^{2}\right\rangle=\int \operatorname{PDF}(u, v, x, y) \cdot u^{\prime 2} \mathrm{~d} u \mathrm{~d} v \\
& \left\langle v^{\prime}(x, y)^{2}\right\rangle=\int \operatorname{PDF}(u, v, x, y) \cdot v^{\prime 2} \mathrm{~d} u \mathrm{~d} v
\end{aligned}
$$

and

$$
\begin{aligned}
\left\langle u(x, y)^{\prime} \cdot v(x, y)^{\prime}\right\rangle & =\left\langle v(x, y)^{\prime} \cdot u(x, y)^{\prime}\right\rangle \\
& =\int \operatorname{PDF}(u, v, x, y) \cdot u^{\prime} \cdot v^{\prime} \mathrm{d} u \mathrm{~d} v .
\end{aligned}
$$

The integration limits are $-\infty$ or $+\infty$, respectively. Equations $2 \mathrm{a}-\mathrm{c}$ must now be multiplied by the mass density in order to provide the actual Reynolds stresses. For simplicity, the expected values of the fluctuation products will be identified as Reynolds stresses in this paper. Equations $2 \mathrm{a}-\mathrm{c}$ are valid for any normalized PDF, hence the challenge that remains lies in solving the integrals. Furthermore, the most important task is to identify the probability density function from the correlation peak. Therefore, the shape of the correlation peak, as a function of the PDF, will be analyzed in the following.

\subsection{A general analytical expression of the correlation function}

In the following analysis, the variables for the position vector and other quantities are used on both, the measurement plane in physical space and the image plane (camera sensor). Lower case letters $(x, y, \ldots)$ refer to quantities on the measurement plane, and upper case letters $(X, Y, \ldots)$ denote quantities on the image plane. The position vectors on the two planes are directly related via the magnification $M$ of the imaging system and the pixel size $S$ of the camera sensor:

$$
(X, Y)=(x, y) \cdot M / S \text {. }
$$

The velocity on the measurement plane is transferred into the shift vector on the image plane: 
$(\Delta X, \Delta Y)=(u, v) \cdot \Delta t \cdot M / S$

Table 1 summarizes the most important variables used in the theoretical discussion.

For an ensemble correlation with single-pixel resolution, the correlation function $C(\xi, \psi, X, Y)$ can be computed from a pair of PIV images $A(X, Y)$ and $B(X, Y)$ as follows:

$$
\begin{aligned}
C(\xi, \psi, X, Y)= & \sum_{n}\left\{\left[A_{n}(X, Y)-\bar{A}(X, Y)\right]\right. \\
& \left.\cdot\left[B_{n}(X+\xi, Y+\psi)-\bar{B}(X+\xi, Y+\psi)\right]\right\} / \\
& \{\sigma A(X, Y) \cdot \sigma B(X+\xi, Y+\psi)\}
\end{aligned}
$$

where the standard deviation is given by:

$\sigma A(X, Y)=\sqrt{\frac{1}{N-1} \sum_{n}\left[A_{n}(X, Y)-\bar{A}(X, Y)\right]^{2}}$

and $N$ and $n$ are the total number of PIV image pairs and the corresponding control variable. $(X, Y)$ are discrete coordinates of the pixel in question in both images, and $(\xi, \psi)$ are the coordinates on the correlation plane.

In order to analyze the shape of the correlation peak for a fixed point $(X, Y)$ in the image plane, Eq. 5 is converted into an analytical expression.

Table 1 Frequently used variables and their meaning

\begin{tabular}{lll}
\hline Quantity & Symbol & Unit \\
\hline Coordinates on measurement plane & $x$ & $\mathrm{~m}$ \\
Coordinates on image plane & $y$ & $\mathrm{~m}$ \\
Velocity components on measurement plane & $X$ & $\mathrm{px}$ \\
& $Y$ & $\mathrm{px}$ \\
Coordinates on correlation plane & $v$ & $\mathrm{~m} / \mathrm{s}$ \\
& $\xi$ & $\mathrm{m} / \mathrm{s}$ \\
Shift vector components & $\psi$ & $\mathrm{px}$ \\
& $\Delta X$ & $\mathrm{px}$ \\
Parameters of PDF on measurement plane & $p_{x}$ & $\mathrm{px}$ \\
& $p_{y}$ & $\mathrm{px}$ \\
& $\alpha$ & $\mathrm{m} / \mathrm{s}$ \\
Parameters of PDF on image plane & $P_{X}$ & $\mathrm{rad}$ \\
Particle image diameter & $P_{Y}$ & $\mathrm{px}$ \\
Magnification & $\alpha$ & $\mathrm{rad}$ \\
Pixel size & $C_{X}$ & $\mathrm{px}$ \\
& $C_{Y}$ & $\mathrm{px}$ \\
Parameter of correlation peak & $\varphi$ & $\mathrm{rad}$ \\
& $D$ & $\mathrm{px}$ \\
& $M$ & $\mathrm{~m} / \mathrm{m}$ \\
& $S$ & $\mathrm{~m} / \mathrm{px}$ \\
& &
\end{tabular}

A cross-correlation integral of the two images $A$ and $B$ replaces the sum in Eq. 5. The discrete images $A_{n}$ are replaced by one continuous Gaussian peak representing the particle image intensity distribution:

$A_{n}(X, Y) \rightarrow A(X, Y)=\exp \left(-\frac{X^{2}+Y^{2}}{D^{2}} \cdot 8\right)$.

The parameter $D$ in Eq. 6 is the particle image diameter at $1 / e^{2}$ of the maximum intensity. Although the Gaussian intensity profile is a good approximation (Born and Wolf 2000), the derivation can also be based on any other shape, if necessary. However, finding an analytical expression for the correlation function might become more difficult.

The images $B_{n}$ are also replaced by a single Gaussian peak, but two things are different compared to $A_{n}$ : first, they are shifted due to the particle mean motion and secondly, they are convolved (denoted by *) with the probability density function $\operatorname{PDF}(X, Y)$ of the velocity to include velocity fluctuations:

$B_{n}(X, Y) \rightarrow B(X-\Delta X(X, Y), Y-\Delta Y(X, Y)) * \operatorname{PDF}(X, Y)$,

with

$$
\begin{aligned}
& B(X-\Delta X(X, Y), Y-\Delta Y(X, Y)) \\
& =\exp \left(-\frac{(X-\Delta X(X, Y))^{2}+(Y-\Delta Y(X, Y))^{2}}{D^{2}} \cdot 8\right) .
\end{aligned}
$$

By using Eqs. 6 and 7, the correlation function from Eq. 5 can be transformed into an analytical expression:

$$
\begin{aligned}
C(\xi, \psi, X, Y) \sim & A(X, Y) \star[B(X, Y) * \operatorname{PDF}(X, Y)] \\
= & \int A(X-\xi, Y-\psi) \\
& \cdot\left[\int B(\mu-\Delta X(\mu, \eta), \eta-\Delta Y(\mu, \eta))\right. \\
& \cdot \operatorname{PDF}(X-\mu, Y-\eta) \mathrm{d} \mu \mathrm{d} \eta] d X d Y,
\end{aligned}
$$

where $\star$ denotes the cross-correlation and $*$ the convolution. In Eq. 8, the sum of Eq. 5 was replaced by an integral. This ensures that all possible locations of the particle image, with respect to the point of interest, were considered and simulates a perfectly uniform particle image distribution. $\mu$ and $\eta$ are the control variables for the convolution integral. The coordinates $(X, Y)$ are on the image plane, and $(\xi, \psi)$ correspond to the correlation plane. The integration limits are $-\infty$ or $+\infty$, respectively.

Generally, it is only required to compute the theoretical equation for the correlation function and apply this as a fit- 
function to the experimental data in order to identify the probability density function.

\subsection{Reynolds stresses for a specified PDF}

Equation 8 gives a universal expression for a correlation function. However, in order to solve the integral, it is necessary to specify the PDF. The following derivation is made under the assumption that the probability density function $\operatorname{PDF}(X, Y)$ is Gaussian, which is a very good approximation in general. The Gaussian distribution has an elliptical cross-section (major axis $P_{X}$, minor axis $P_{Y}$ ) and is rotated by the angle $\alpha$ :

$$
\begin{aligned}
\operatorname{PDF}(X, Y) & =\frac{8}{\pi \cdot P_{X} \cdot P_{Y}} \\
& \cdot \exp \left[-\left(\frac{\cos \alpha \cdot(X-\Delta X)-\sin \alpha \cdot(Y-\Delta Y)}{P_{X}}\right)^{2} \cdot 8\right. \\
& \left.-\left(\frac{\sin \alpha \cdot(X-\Delta X)+\cos \alpha \cdot(Y-\Delta Y)}{P_{Y}}\right)^{2} \cdot 8\right]
\end{aligned}
$$

The factor in front of the exponential term in Eq. 9 ensures that the integral of the PDF over all velocities is always equal one. The shape of the probability density function can generally change for each point of the image plane, hence $P_{X}, P_{Y}$, and $\alpha$ are functions of the position vector $(X, Y)$. The image plane parameters $P_{X}$ and $P_{Y}$ are derived from the equivalent quantities in the measurement plane as follows:

$\left(P_{X}, P_{Y}\right)=\left(p_{x}, p_{y}\right) \cdot \Delta t \cdot M / S$.

Solving Eq. 8 for the defined particle images (Eq. 6) and the specified PDF from Eq. 9 gives an analytical expression for the correlation function:

$$
\begin{aligned}
C(\xi, \psi, X, Y) & \sim \exp \left[-\left(\frac{\cos \varphi \cdot(\xi-\Delta X)-\sin \varphi \cdot(\psi-\Delta Y)}{C_{X}}\right)^{2} \cdot 8\right. \\
& \left.-\left(\frac{\sin \varphi \cdot(\xi-\Delta X)+\cos \varphi \cdot(\psi-\Delta Y)}{C_{Y}}\right)^{2} \cdot 8\right] .
\end{aligned}
$$

The parameters $C_{X}, C_{Y}$, and $\varphi$ in Eq. 11 are functions of the position vector $(X, Y)$. For a Gaussian PDF, the correlation function is also Gaussian. The elliptical cross-section is enlarged due to the correlation procedure, but it has the same orientation as the PDF for the case without velocity gradients:

$\varphi=\alpha$.

$C_{X}$ and $C_{Y}$ depend on the parameters $P_{X}, P_{Y}$ of the PDF as follows:
$C_{X}=\sqrt{P_{X}^{2}+2 \cdot D^{2}}$

$C_{Y}=\sqrt{P_{Y}^{2}+2 \cdot D^{2}}$

where $D$ is the particle image diameter. Equations $11-13 \mathrm{~b}$ are only exact solutions for the case without velocity gradients. The velocity is assumed to be constant in the surroundings of the point of interest.

Now that the PDF is estimated from the correlation peak, the Reynolds stresses can be calculated from Eqs. 2a-c using Eqs. 9 and 10:

$$
\begin{aligned}
& \left\langle u^{\prime 2}\right\rangle=\frac{1}{16} \cdot\left(\cos ^{2} \alpha \cdot p_{x}^{2}+\sin ^{2} \alpha \cdot p_{y}^{2}\right), \\
& \left\langle v^{\prime 2}\right\rangle=\frac{1}{16} \cdot\left(\sin ^{2} \alpha \cdot p_{x}^{2}+\cos ^{2} \alpha \cdot p_{y}^{2}\right),
\end{aligned}
$$

and

$$
\left\langle u^{\prime} \cdot v^{\prime}\right\rangle=\left\langle v^{\prime} \cdot u^{\prime}\right\rangle=\frac{1}{16} \cdot \cos \alpha \cdot \sin \alpha\left(p_{y}^{2}-p_{x}^{2}\right) .
$$

In the case of isotropic stresses, the minor and major axes are equal $\left(p_{x}=p_{y}\right)$ and the shear stress vanishes along with the dependence on the angle $\alpha$ of all stresses.

The Reynolds stresses on the image plane are directly related to the stresses on the measurement plane via the time $\Delta t$, the magnification $M$, and the pixel size $S$ :

$$
\begin{aligned}
& \left(\left\langle U^{\prime 2}\right\rangle,\left\langle V^{\prime 2}\right\rangle,\left\langle U^{\prime} \cdot V^{\prime}\right\rangle\right) \\
& \quad=\left(\left\langle u^{\prime 2}\right\rangle,\left\langle v^{\prime 2}\right\rangle,\left\langle u^{\prime} \cdot v^{\prime}\right\rangle\right) \cdot\left(\frac{\Delta t \cdot M}{S}\right)^{2} .
\end{aligned}
$$

\subsection{Effect of velocity gradients}

Keane and Adrian reported an analysis of correlation peaks and their dependency on velocity gradients in conventional PIV (Keane and Adrian 1990). They found that for a shear layer the amplitude decreases with increasing velocity gradients and that the diameter is broadened (by the same factor) in the direction of shear. This section investigates the shape of the correlation peak computed with two-point ensemble correlation in a similar manner.

In order to analyze the influence of the velocity gradient on the shape of the correlation function, it is essential to find an expression for the velocity in the area surrounding the point of interest.

Since in many cases, the mean flow has a preferred orientation and strong gradients occur only in the perpendicular direction, it seems to be a good approach to develop the velocity in a Taylor series and neglect all higher terms except the first derivative of $u$ with respect to $y$. For the shift vector on the image plane, this procedure results in the following expression: 


$$
\Delta \mathbf{X}\left(X_{0}, Y_{0}\right) \approx\left(\begin{array}{c}
\Delta X\left(X_{0}, Y_{0}\right)+\left.Y \cdot \frac{\partial \Delta X(X, Y)}{\partial Y}\right|_{\left(X_{0}, Y_{0}\right)} \\
\Delta Y\left(X_{0}, Y_{0}\right)
\end{array}\right) .
$$

where $\left(X_{0}, Y_{0}\right)$ is the point of interest on the image plane for which the correlation function has to be computed.

Taking this velocity gradient into account leads to a rather complex solution of Eq. 8:

$$
\begin{aligned}
C(\xi, \psi, X, Y) \sim & \exp \left\{\left[-32 \cdot\left(P_{X}^{2}+P_{Y}^{2}\right)\right.\right. \\
& \cdot\left\{\psi^{2}+\xi^{2}+\left(1+\Delta X_{Y}^{2}\right) \cdot \Delta Y^{2}\right. \\
& +2 \cdot \Delta X \cdot\left(\Delta X_{Y} \cdot \Delta Y-\xi\right) \\
& \left.-2 \cdot \Delta Y \cdot\left(\psi+\Delta X_{Y} \cdot \xi\right)+\Delta X^{2}\right\} \\
+ & 2 \cdot D^{2} \cdot\left\{2 \cdot \xi^{2}+2 \cdot \Delta X^{2}\right. \\
& +\left(2+\Delta X_{Y}^{2}\right) \cdot \psi^{2}+\left(2+\Delta X_{Y}^{2}\right) \cdot \Delta Y^{2} \\
& +2 \cdot \Delta X \cdot\left[\Delta X_{Y} \cdot(\psi+\Delta Y)-2 \cdot \xi\right] \\
& -2 \cdot \Delta Y \cdot\left(2 \cdot \psi+\Delta X_{Y} \cdot \xi\right) \\
& \left.-2 \cdot \Delta X_{Y} \cdot \xi \cdot \psi\right\} \\
+ & \left(P_{X}-P_{Y}\right) \cdot\left(P_{X}+P_{Y}\right) \\
& \cdot\left\{\left[\psi-\xi+\left(\Delta X_{Y}-1\right) \cdot \Delta Y+\Delta X\right]\right. \\
& \cdot\left[\xi+\psi-\left(\Delta X_{Y}+1\right) \cdot \Delta Y-\Delta X\right] \\
& \cdot \cos (2 \cdot \alpha)+2 \cdot\left(\Delta X_{Y} \cdot \Delta Y+\Delta X-\xi\right) \\
& \cdot(\Delta Y-\psi) \cdot \sin (2 \cdot \alpha)\}] / \\
& \left\{2 \cdot D ^ { 2 } \cdot \left[D^{2} \cdot\left(16+\Delta X_{Y}^{2}\right)+\left(P_{X}^{2}+P_{Y}^{2}\right)\right.\right. \\
& \left.\cdot\left(4+\Delta X_{Y}^{2}\right)\right]+6 \cdot D^{4} \cdot \Delta X_{Y}^{2}+8 \cdot P_{X}^{2} \cdot P_{Y}^{2} \\
+ & 2 \cdot D^{2} \cdot\left(P_{X}^{2}+P_{Y}^{2}\right) \cdot\left(4+\Delta X_{Y}^{2}\right) \\
- & 4 \cdot D^{2} \cdot\left(P_{X}-P_{Y}\right) \cdot\left(P_{X}+P_{Y}\right) \cdot \Delta X_{Y} \\
& \left.\left.\cdot\left(\Delta X_{Y} \cdot \operatorname{Cos}(2 \cdot \alpha)-2 \cdot \sin (2 \cdot \alpha)\right)\right\}\right\} .
\end{aligned}
$$

The term $\Delta X_{Y}$ in Eq. 17 identifies the velocity gradient (or more precisely the shift vector gradient) $\partial \Delta X / \partial Y . P_{X}, P_{Y}$, and $\alpha$ are the parameters of the probability density function on the image plane from Eq. 9 .

Again, Eq. 17 is Gaussian with an elliptical cross-section. However, the minor and major axis and the angle of rotation can not be extracted from the equation as easily as before.

Figure 1 shows the correlation function from Eq. 17 for different conditions. The PDF was non-isotropic $\left(P_{X}=5 \mathrm{px}, P_{Y}=1 \mathrm{px}\right)$ in all cases and the angle of orientation was $\alpha=20^{\circ}$. A particle image diameter $D$ of 1,3 , and $5 \mathrm{px}$ was used for the left, middle, and right column, respectively.

No gradient $\partial \Delta X / \partial Y$ was applied for the middle row. Here, the resulting correlation peaks look similar to the applied PDF (cross-section shown as red solid line), and Eqs. 13a and $\mathrm{b}$ are sufficient to determine the parameters of the PDF.
The upper and lower rows in Fig. 1 show the influence of an additional velocity gradient $\partial \Delta X / \partial Y$. Here, the correlation peaks are stretched in the direction of $P_{X}$ according to the analysis of Keane and Adrian (1990) but, in addition, they are compressed in the direction of $P_{Y}$ and rotated around the center due to the velocity gradient. The rotation direction depends on the sign of the velocity gradient.

Consequently, it is important to note that the shape of the PDF is not directly related to the shape of the correlation function in the case of strong velocity gradients. Therefore, it is essential to include the information about the surrounding velocity field in order to compute the Reynolds stresses for a given point (Kähler and Scholz 2006).

Using Eqs. 13a and $b$ for the reconstruction of the PDF and the estimation of the Reynolds stresses leads to a systematic error in the presence of velocity gradients. As a result of this, the normal stress in the $X$-direction is overestimated and the Reynolds shear stress is under or overestimated, depending on the orientation of the PDF and the sign of the velocity gradient.

Additionally, Fig. 1 shows that the influence of velocity gradients on the shape of the correlation function is strongly dependent on the size of the particle images. The deformation gets stronger with increasing particle image diameter $D$. The compensation of the velocity gradient effects will be discussed in Sect. 3.5.

The broadening of the correlation peaks under the influence of strong velocity gradients is in qualitative agreement with the considerations for conventional PIV made by Keane and Adrian (1990) as well as Kähler and Scholz (2006).

\section{Analysis of synthetic PIV images}

In order to determine the accuracy of the method presented previously, synthetic PIV images with different probability density functions were generated and the estimated Reynolds stresses were compared to the simulated values. This allows for a quantitative accuracy assessment.

The calculation procedure is described in Sect. 3.1. Section 3.2 deals with isotropic stresses and investigates the effect of several parameters on the accuracy of the estimated turbulence. Section 3.3 focuses on the more general case of homogeneous non-isotropic Reynolds stresses. The ability to estimate shear stresses as well as the demonstration of the presented method's spatial resolution are analyzed in Sect. 3.4. The last section of this chapter studies velocity gradient effects and their compensation (Sect. 3.5). 
Fig. 1 Contour plot of the analytical correlation function for different velocity gradients $\partial \Delta X / \partial Y([1,0,-1] \mathrm{px} / \mathrm{px}$ from top to bottom) and different particle image diameters $D$ $([1,3,5] \mathrm{px}$, from left to right $)$. The size and orientation of the probability density function is indicated by the solid red line, the corresponding parameters $P_{X}, P_{Y}$, and $\alpha$ are 5, $1 \mathrm{px}$, and $20^{\circ}$, respectively
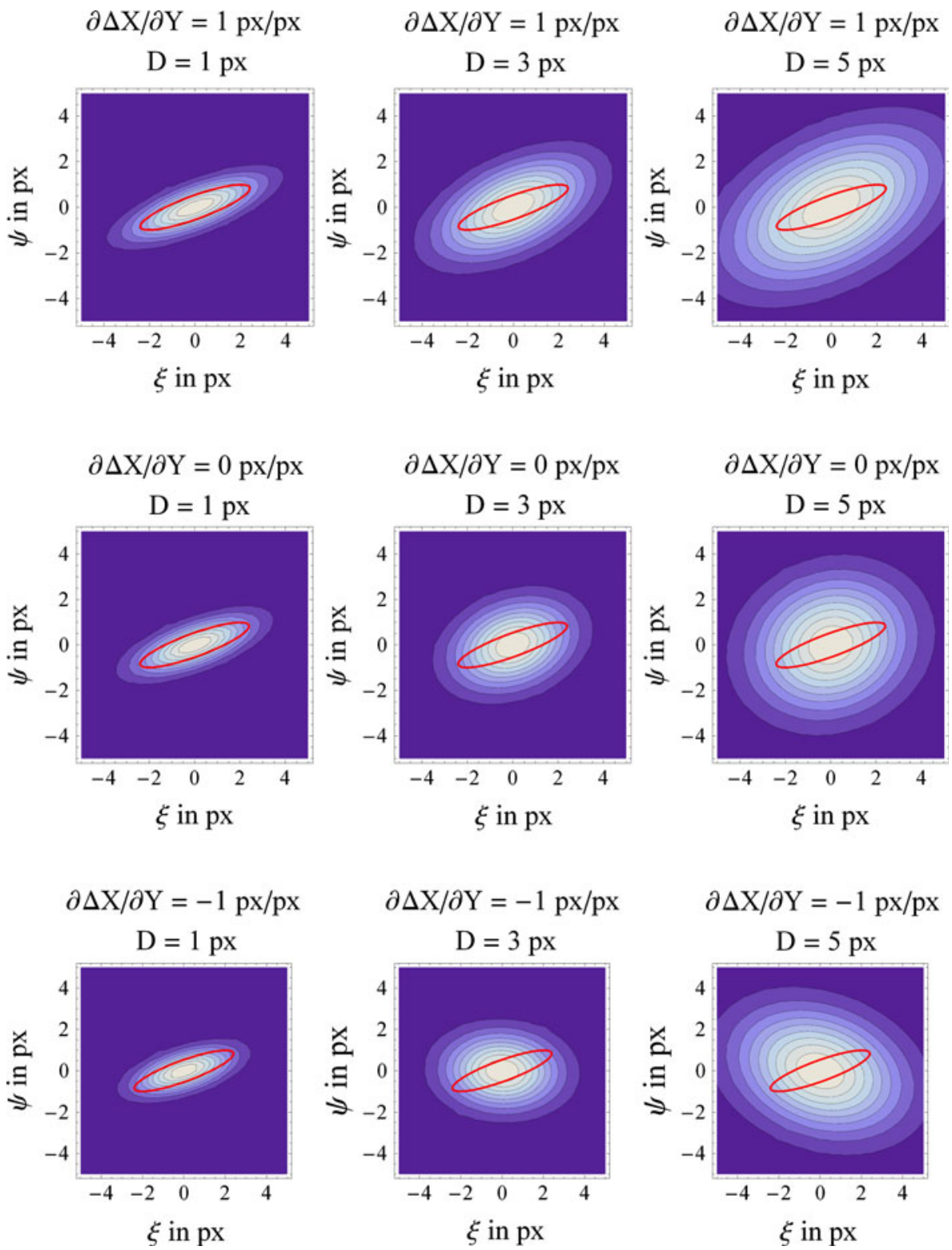

\subsection{Calculation procedure}

All synthetic PIV images were generated and analyzed using MatLab functions. Unless otherwise stated, each single test case consists of 20,000 PIV image pairs. The size of the images is such that the correlation function was computed for $20 \mathrm{px} \times 20 \mathrm{px}$ (a wide margin of several pixel is required for the calculation process).

A maximum of 20,000 counts was applied for the intensity of the particle images (Gaussian intensity distribution). Each pixel's gray value is computed from the integral of the intensity over the corresponding area, with respect to the finite pixel size of the camera sensor, instead of simply transferring the analytical point-wise values to the pixel. This procedure causes a difference between the applied particle image diameter $D_{\text {simulated }}$ and the resulting diameter computed with a Gaussian fit-function $D_{\text {estimated. }}$ Additionally, a normally distributed noise with a half-width of 200 counts was added to each individual image, which results in a signal-to-noise ratio of 100 .

The fraction of illuminated pixels was kept constant at $10 \%$ for all generated images, meaning that $10 \%$ of all pixels had an intensity of more than 20,000 counts/ $e^{2} \approx 2,700$ counts. Hence, the number of particle images changes with the particle image diameter. The average number of particle images in a $20 \mathrm{px} \times 20 \mathrm{px}$ image is, for example, 5.6 for a particle image diameter of $D=3$ px. 
For each particle in each first PIV image, the position is randomly chosen. The position in the second image changed with respect to the first one due to the velocity and its fluctuations. The vertical component of the velocity was zero, while a constant gradient was used for the horizontal component $(\partial \Delta X / \partial Y=0.05)$. The velocity fluctuations were random numbers with a Gaussian distribution. However, the amplitude of the fluctuations was controlled by the parameters $P_{X}, P_{Y}$, and $\alpha$ of the PDF. The theoretical Reynolds stresses were computed from the applied fluctuations.

Upon the generation of the synthetic PIV images, the correlation function for each pixel is computed using Eq. 5. In order to determine the particle image diameter $D$, the autocorrelation function is also required (Kähler and Scholz 2006). The latter can be computed by using Eq. 5 and simply exchanging image $B$ with image $A$. For better accuracy, $D$ is also estimated from the autocorrelation of the $B$ images. In theory, the autocorrelation peak of a Gaussian function is also a Gaussian function, but the width is increased by a factor of $\sqrt{2}$ (Raffel et al. 2007). Hence, fitting the autocorrelation peak gives an estimation of the particle image diameter. For experimental PIV recordings, it was found that the particle images can be stretched in case of supersonic or hypersonic flow. Hence, the autocorrelation peaks should be compared to a Gaussian with elliptical cross-section.

Figure 2 shows the comparison between the input value and the estimated diameter. The proportionality only holds for particle images bigger than $\approx 3 \mathrm{px}$, whereas for smaller values the particle image size is estimated to be too large. The deviation can be corrected using the fit-function in Fig. 2.

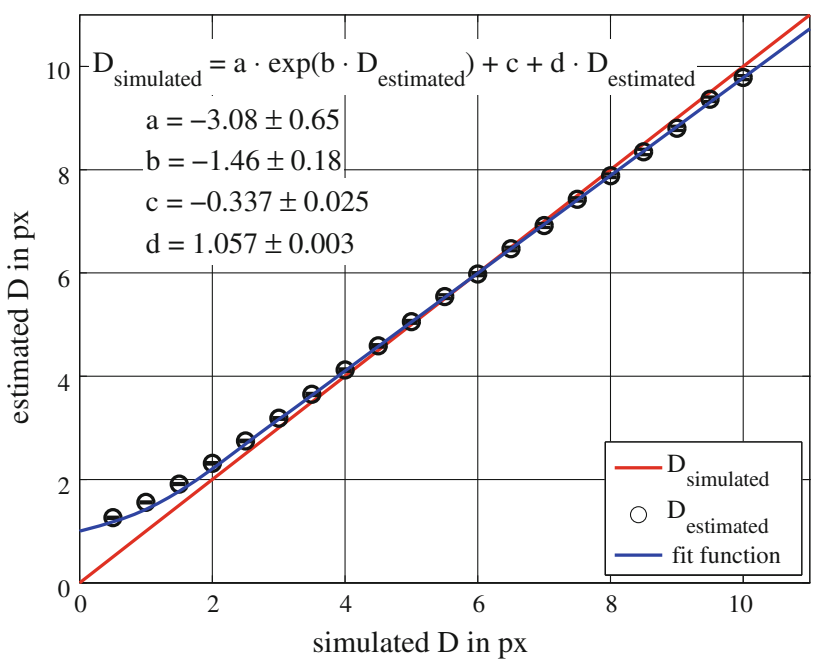

Fig. 2 Estimation of the particle image diameter $D$ for each 20,000 synthetic PIV image pairs. The graph shows the mean value over $20 \mathrm{px} \times 20 \mathrm{px}$ and the corresponding standard deviation as error-bar
A Gaussian fit-function with the same structure as Eq. 11 was applied to the correlation peaks in the next step. With this, the parameters $C_{X}, C_{Y}$, and $\varphi$ of each correlation function were determined. The estimation of the correlation peak's diameter is also subject to a systematic error, as shown in Fig. 3 for the major axis $C_{X}$.

Both particle image diameter and correlation peak diameter were corrected by using the fit-function shown in Figs. 2 and 3, respectively.

The parameters $P_{X}$ and $P_{Y}$ of the PDF were computed by using Eqs. 13a and b, and finally the Reynolds stresses were determined from Eqs. 14a-c. The compensation of velocity gradient effects is discussed in detail in Sect. 3.5.

\subsection{Isotropic stresses}

In this section, PIV images with isotropic stress distribution are evaluated. Isotropic stress means that the parameters $P_{X}$ and $P_{Y}$ are equal and Eq. 9 becomes:

$\operatorname{PDF}\left(X, Y, P_{X}=P_{Y}\right)$

$$
=\frac{8}{\pi \cdot P_{X}^{2}} \exp \left[-\frac{(X-\Delta X)^{2}+(Y-\Delta Y)^{2}}{P_{X}^{2}} \cdot 8\right] \text {. }
$$

In this case, the parameters $C_{X}$ and $C_{Y}$ of the fit-function (Eq. 11) should also be equal. Thus, a symmetric fitfunction can be applied.

A turbulent flow with isotropic stresses has a rotationally symmetric PDF and is thus free of shear stresses. In a laminar flow, the Brownian motion of the tracer particles causes a broadening of the correlation peak. This can be treated as isotropic stresses to determine the temperature

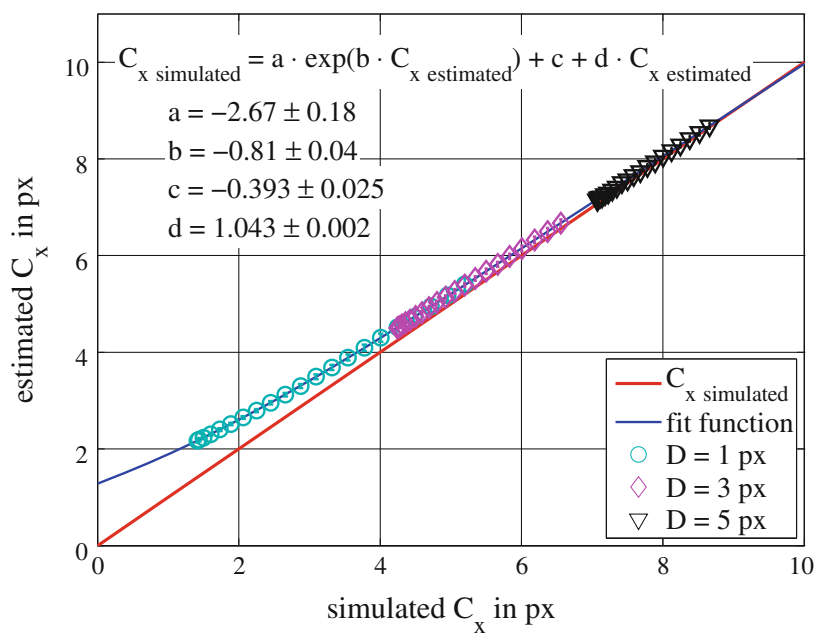

Fig. 3 Estimation of the correlation peak diameter $C_{x}$ for each 20,000 synthetic PIV image pairs. The graph shows the mean value over $20 \mathrm{px} \times 20 \mathrm{px}$ and the corresponding standard deviation as error-bar 
within the flow in a non-intrusive way. This was demonstrated by Hohreiter et al. (2002) and Chamarthy et al. (2009) for correlation peaks computed from interrogation windows with standard PIV evaluation.

First, the influence of the particle image size on the accuracy of the determination of the correlation peak's size and position was investigated. Figure 4 shows the RMSuncertainty of the estimated correlation peak diameter $C_{X}$ with respect to the particle image diameter $D$ for four different values of $P_{X}$. Each point in Figs. 4, 5 and 6 represents the standard deviation of $20 \times 20$ correlation peaks computed from 20,000 synthetic PIV image pairs using Eq. 5. For the generation of the synthetic PIV images, the applied probability density function was constant for all pixel in the image plane.

All four curves in Fig. 4 show a local minimum for a certain particle image diameter, depending on the amplitude of the velocity fluctuations. The parameter $P_{X}$, in real measurements, not only depends on the turbulence in the flow but also on the time $\Delta t$ between the acquisition of the two PIV images, the magnification $M$, and the pixel size $S$ and can thus be partially controlled by these parameters.

As an example, the investigation of the wake flow of a blunt axis-symmetric space launcher model in Bitter et al. (2010) resulted in velocity fluctuations with a maximum of $P_{X}=4.5 \mathrm{px}$ in the shear layer of the wake at $\mathrm{Ma}=0.7$, while the maximum value in the turbulent boundary layer was around $P_{X}=1.5 \mathrm{px}$.

Figure 4 shows a minimum error for a particle image diameter of $D \approx 1.8 \mathrm{px}$ without velocity fluctuations. This minimum shifts to larger values of $D$ for increasing fluctuations. Additionally, the minimum RMS-uncertainty increases. The fact that the random error increases consistently for larger particle images can mainly be explained

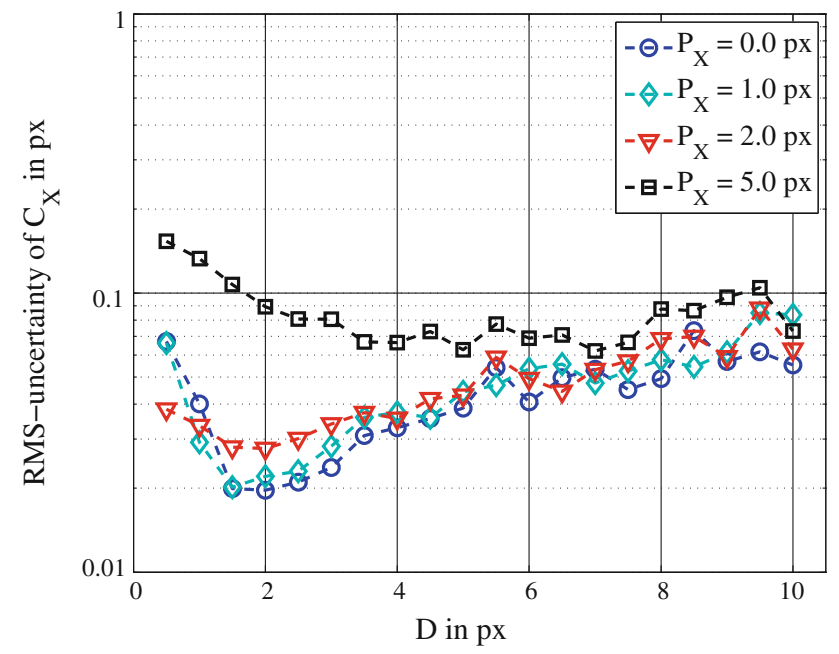

Fig. 4 Influence of the particle image diameter $D$ on the accuracy of the estimated size of the correlation peak $C_{X}$ by the decreasing number of particle images (the fraction of illuminated pixels was kept constant at $10 \%$ ).

The maximum position of the correlation peak represents the mean velocity and is estimated by using a threepoint Gauss-fit. The accuracy of the estimated velocity is shown in Fig. 5. Particle images smaller than $D \approx 1 \mathrm{px}$ are not suitable for accurate velocity measurements, they cause so-called peak locking. This is mostly visible by using a histogram plot as displayed first in Kähler (1997). For particle images larger than $D \approx 2 \mathrm{px}$, the RMSuncertainty remains nearly constant and even drops slightly. This differs from the results presented by Raffel et al. (2007) for single-pass window correlation, where a global minimum error for $D \approx 1.8$ px was found. Overall,

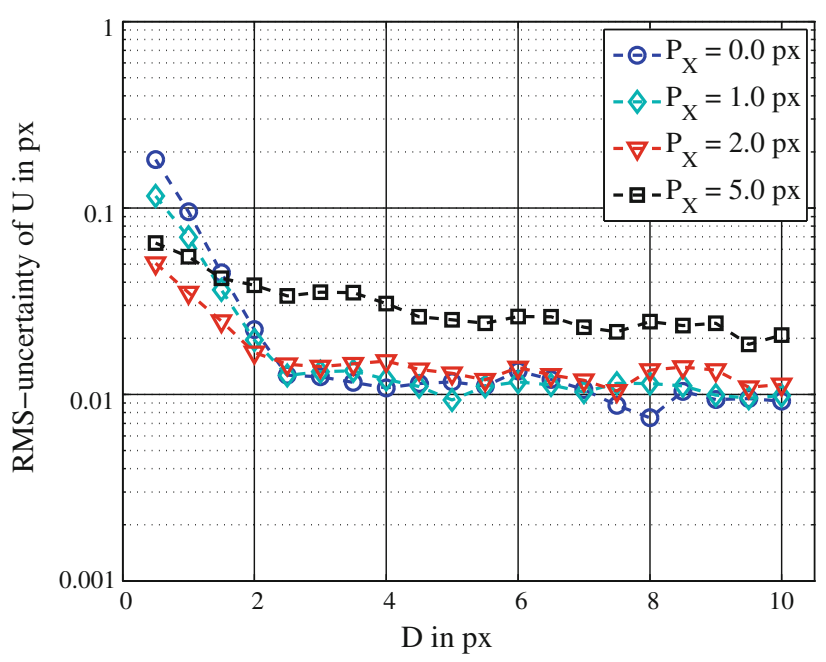

Fig. 5 Influence of the particle image diameter $D$ on the accuracy of the computed velocity component $U$

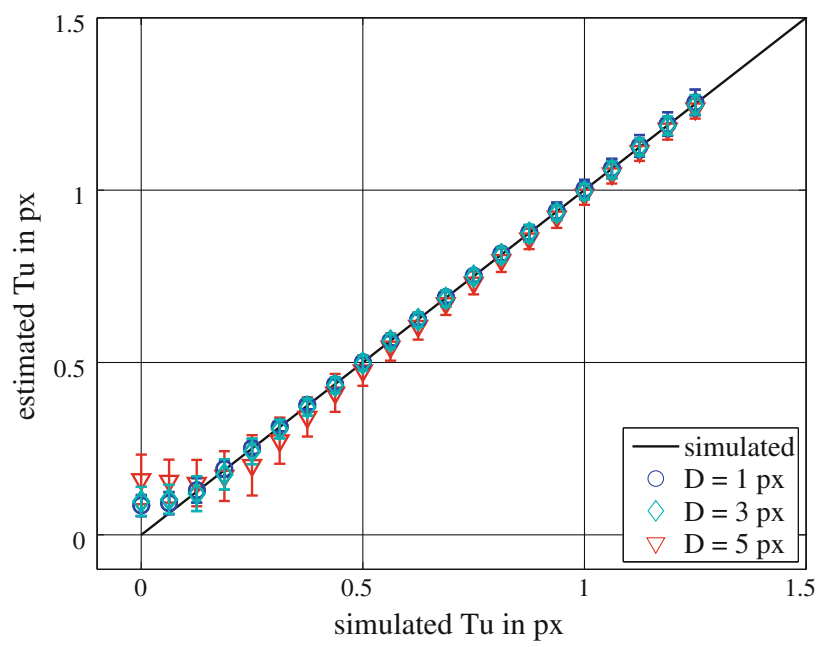

Fig. 6 Systematic error of the estimated turbulence level $T u$ depending on the applied turbulence 
the minimum error of about $0.01 \mathrm{px}$ is comparable to that obtained by using standard PIV methods.

The optimum particle image diameter seems to be around $D=3$ px. At this size, the uncertainty for the estimation of correlation peak diameter and its maximum position (or mean velocity) are fairly low and the particle image size can be well estimated.

The parameter $P_{X}$ of the PDF can be determined from the correlation peak size and the particle image diameter by using Eq. 13a, and the normal stresses are computed from Eqs. 14a and b. Based on the two measured velocity components, the turbulence level $T u$ is used to evaluate the computed stresses. It is defined as follows:

$T u=\sqrt{\frac{\left\langle U^{\prime 2}\right\rangle+\left\langle V^{\prime 2}\right\rangle}{2}}$.

Furthermore, with Eqs. 14a and b, this can be reduced to:

$T u=\frac{P_{x}}{4}$

in the case of isotropic stresses.

Figure 6 shows the estimated turbulence level with respect to the simulated turbulence level for three different particle image diameters. For small fluctuations, the correlation and autocorrelation peaks have almost the same diameter. Since the PDF's diameter is computed from their difference (Eqs. 13a, b), its error grows considerably in this case. However, for the precise determination of small Reynolds stresses, the time $\Delta t$ between the two PIV images, and thus the correlation peak size, can be increased. For a turbulence level larger than $T u>0.2 \mathrm{px}$, the systematic error becomes negligible. For the real example of the round jet in Sect. 4.1, the turbulence level was between $T u=0.1 \mathrm{px}$ (undisturbed flow) and $T u=1.5 \mathrm{px}$ (in the shear layer). For the wake flow in Sect. 4.2, it was between $T u=0.25$ px (in the boundary layer) and $T u=1.3 \mathrm{px}$ (in the shear layer).

The accuracy of the estimated turbulence level is not only dependent on the particle image diameter and the turbulence level itself but also on the number of image pairs. Figures 7 and 8 illustrate how the number of PIV images $N$ affects the estimated turbulence and mean velocity, respectively. The fraction of illuminated pixels was still $10 \%$. As expected, the error decreases with an increasing number of images. For the tested cases, 10,000 image pairs appear to be a sufficient number.

\subsection{Homogeneous flow with non-isotropic stresses}

So far, only synthetic PIV images with symmetric probability density functions were analyzed. In the following, the more general approach from Eq. 9 will be applied. Thus, the normal stresses $\left\langle U^{\prime 2}\right\rangle$ and $\left\langle V^{\prime 2}\right\rangle$ are not necessarily equal and the shear stress $\left\langle U^{\prime} \cdot V^{\prime}\right\rangle$ can differ from zero.

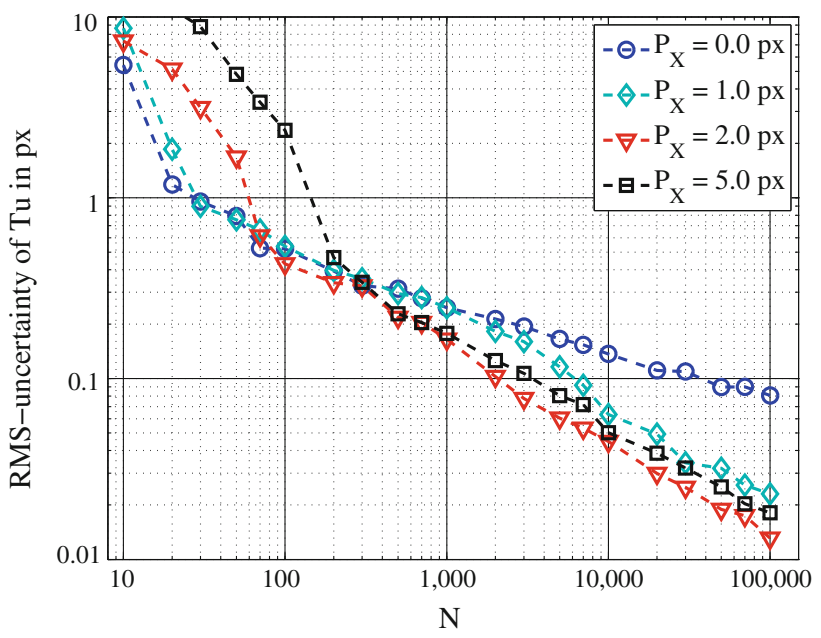

Fig. 7 Influence of the number of synthetic PIV images on the accuracy of the estimated turbulence level $T u$. The particle image diameter was $D=3 \mathrm{px}$

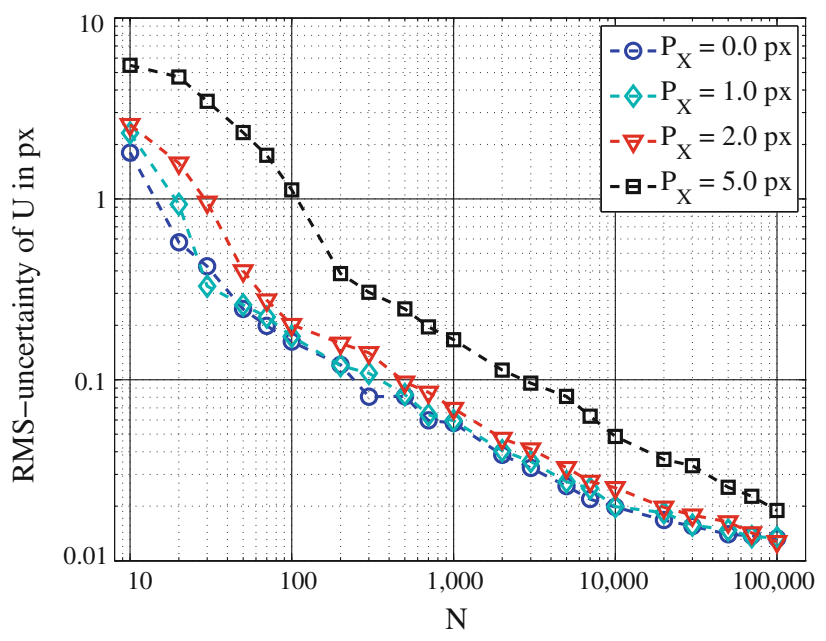

Fig. 8 Influence of the number of synthetic PIV images on the accuracy of the estimated mean velocity. The particle image diameter was $D=3 \mathrm{px}$

However, the simulated stresses are still constant over the whole image plane.

Figure 9 shows the RMS-uncertainty for the estimated Reynolds shear stress with respect to the number of image pairs $N$. The parameters $P_{X}, P_{Y}$ of the PDF were fixed at 2 and $0.5 \mathrm{px}$, respectively; while four different angles $\alpha$ were tested. The RMS-uncertainty decreases exponentially over $N$.

Figures 10 and 11 show the comparison between the applied and the estimated Reynolds stresses for different

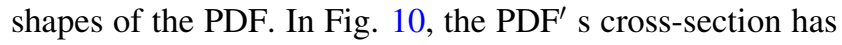
a constant size but is rotated by the angle $\alpha$. The stresses for a constant angle of rotation and increasing ratio of $P_{Y} / P_{X}$ 


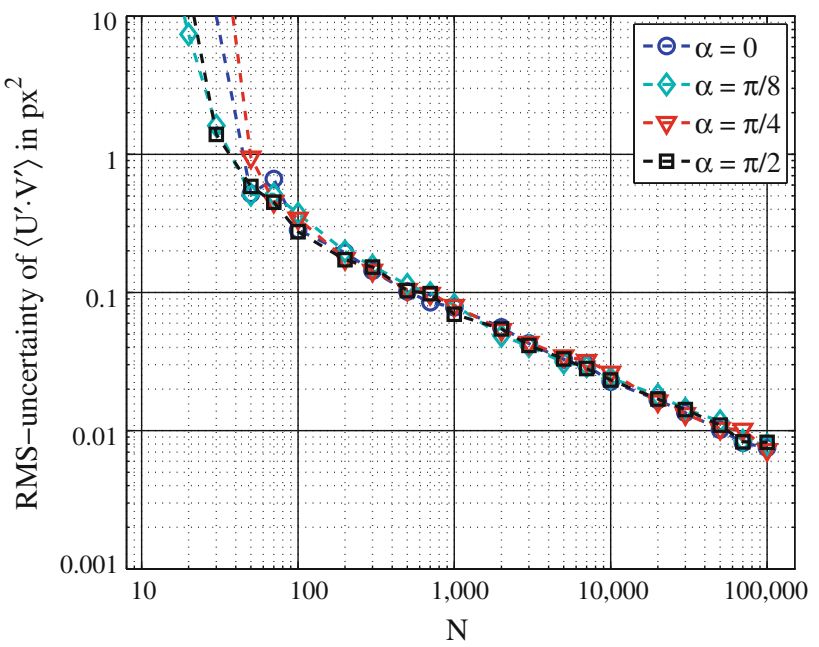

Fig. 9 Influence of the number of synthetic PIV images on the accuracy of the computed velocity. The particle image diameter was $D=3$ px
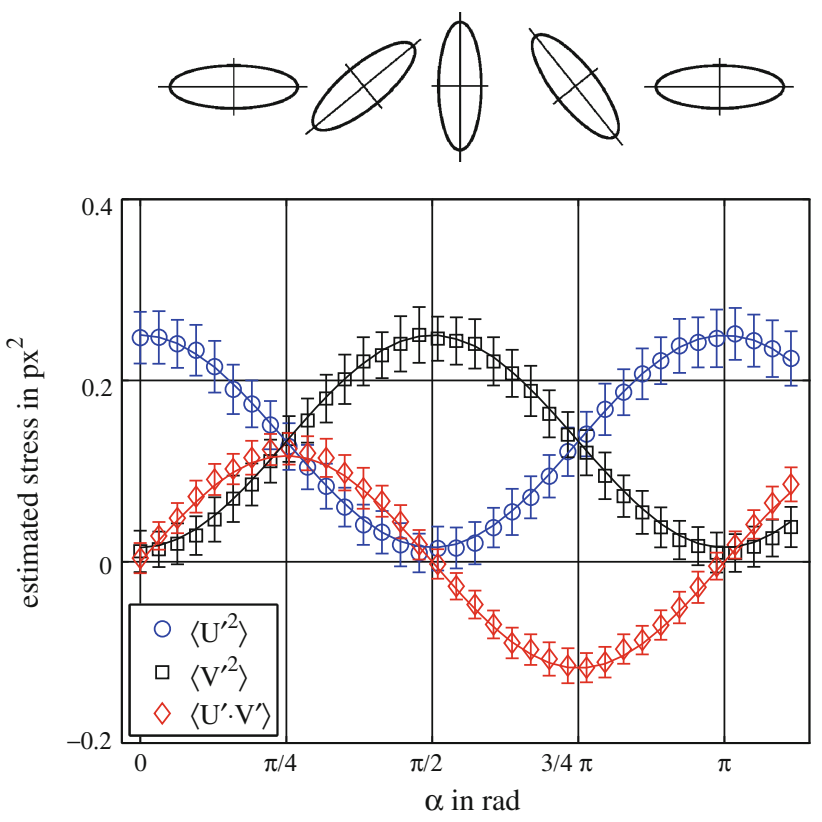

Fig. 10 Estimated Reynolds stresses for 20,000 PIV images $(20 \mathrm{px} \times 20 \mathrm{px})$. The probability density function had a fixed axis ratio $\left(P_{X}=2.0 \mathrm{px}, P_{y}=0.5 \mathrm{px}\right)$ and a varying angle of rotation $(\alpha=0 \ldots \pi)$. A cross-section through the correlation function is sketched in the top part

are shown in Fig. 11. Each point was computed from 20,000 PIV image pairs with constant stress; $20 \times 20$ correlation peaks were analyzed. The particle image diameter was $D=3 \mathrm{px}$. The error-bars correspond to the standard deviation of the stresses for each 400 correlation peaks.

Overall, the estimated mean values for the stresses are in good agreement with the simulated ones. The maximum

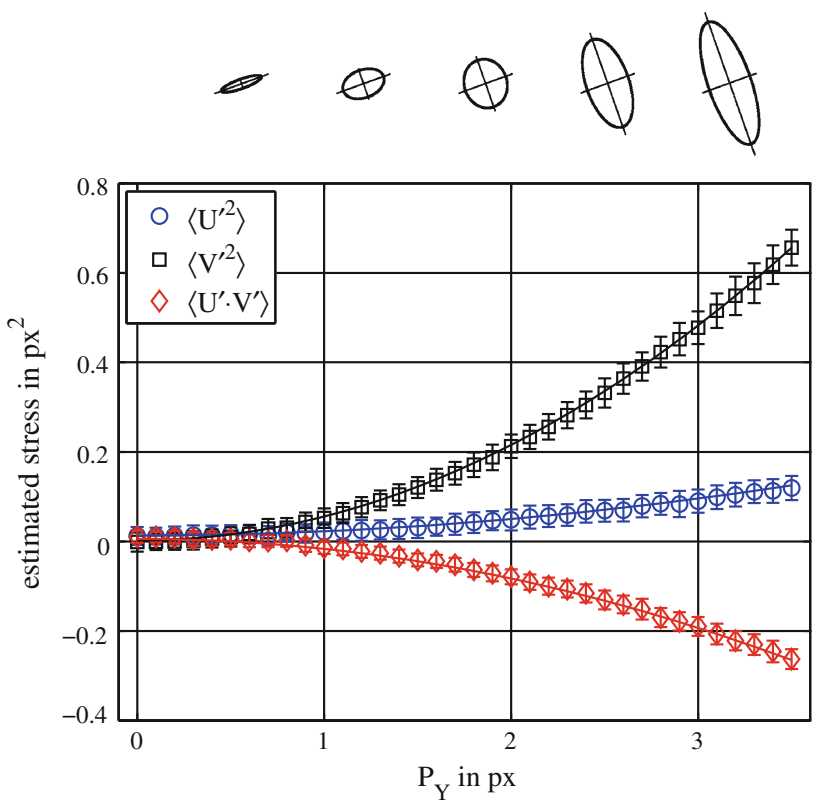

Fig. 11 Estimated Reynolds stresses for 20,000 PIV images $(20 \mathrm{px} \times 20 \mathrm{px})$. The probability density function had a fixed angle of rotation $(\alpha=\pi / 8)$ and a fixed major axis $\left(P_{X}=0.5 \mathrm{px}\right)$, the minor axis $P_{Y}$ varied from 0 to $2 \mathrm{px}$. A cross-section through the correlation function is sketched in the top part

RMS-error is $0.03 \mathrm{px}^{2}$. Further tests with different probability density functions showed similar results.

According to the previous analysis, the developed method is suitable for estimating Reynolds stresses, in particular shear stresses. Although the stresses are computed for each single-pixel, only mean values over $400 \mathrm{px}$ are plotted above. Hence, it cannot be called real singlepixel resolution in this case. However, results with true single-pixel resolution are presented in the next two sections.

\subsection{Shear flow with non-isotropic stresses}

This section demonstrates the enhanced spatial resolution for an example of non-homogeneous Reynolds stresses. Therefore, 100,000 synthetic PIV image pairs, $256 \mathrm{px} \times$ 256 px in size, were generated with a Siemens-star shaped stress distribution.

The mean velocity was kept constant about the whole image area. A particle image diameter of $D=3$ px was applied, and the fraction of illuminated area was set to $2.5 \%$. Hence, on average 0.9 particle images are within a $16 \mathrm{px} \times 16 \mathrm{px}$ window. This corresponds to a relatively low seeding density.

The velocity fluctuations were totally uncorrelated, meaning that even two closely spaced particle images can move in different directions. This simulates small scale turbulent structures. 
Fig. 12 Turbulence level (top) and Reynolds shear stress (bottom) for a synthetic test case of 100,000 PIV recordings. The simulated values are shown in the left column and the computed results using the single-pixel approach and standard window correlation techniques are illustrated in the middle and right column, respectively
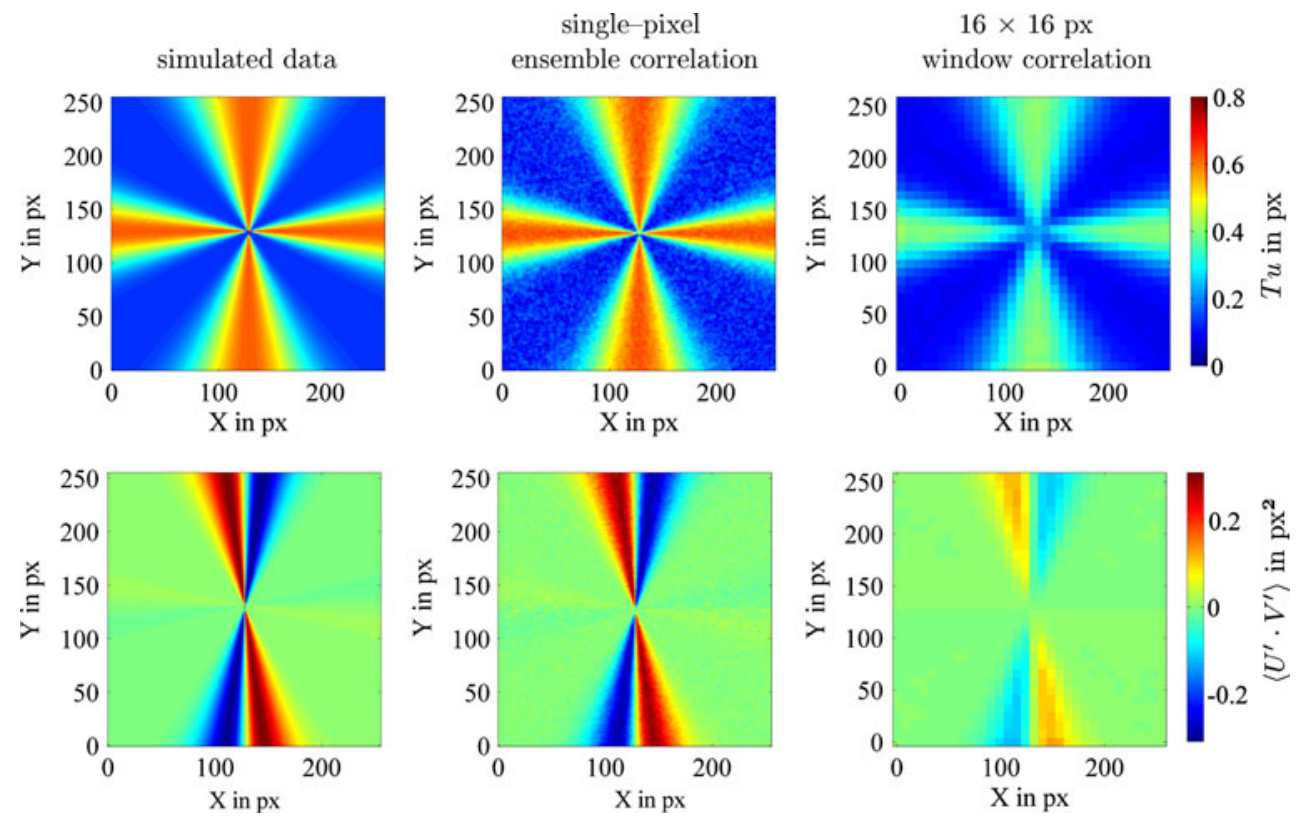

The following parameters $P_{X}, P_{Y}$, and $\alpha$ of the PDF were applied:

$$
\begin{aligned}
P_{X}= & 0.5 \mathrm{px}+3 \mathrm{px} \cdot \exp \left[-8 \cdot\left(\frac{X-128 \mathrm{px}}{|Y-128 \mathrm{px}| \cdot 1.25}\right)^{2}\right] \\
& +1 \mathrm{px} \cdot \exp \left[-8 \cdot\left(\frac{Y-128 \mathrm{px}}{|X-128 \mathrm{px}| \cdot 0.75}\right)^{2}\right], \\
P_{Y}= & 0.5 \mathrm{px}
\end{aligned}
$$

and

$\alpha=\operatorname{sign}(Y-128 \mathrm{px}) \cdot \arctan \left[\frac{(X-128 \mathrm{px}) \cdot 2 \pi}{|Y-128 \mathrm{px}| \cdot 1.25}+\frac{\pi}{2}\right]$.

Figure 12 shows the computed results for the turbulence level $T u$ as defined in Eq. 19 and the Reynolds shear stress $\left\langle U^{\prime} \cdot V^{\prime}\right\rangle$ in the lower and upper row, respectively. The simulated values are plotted in the left column, while the estimations using ensemble correlation with single-pixel resolution and window correlation are shown in the middle and right columns, respectively.

Although the single-pixel results for the turbulence level and shear stress in the middle column of Fig. 12 are somewhat noisy, it is clearly shown that small scale flow phenomena can be resolved. The estimated values are consistent with the simulated ones in the single-pixel approach case.

On the other hand, the values computed with window correlation approach (right column in Fig. 12) do not show such good spatial resolution and are not able to resolve small scale structures. Additionally, the stresses computed with the window correlation method are significantly

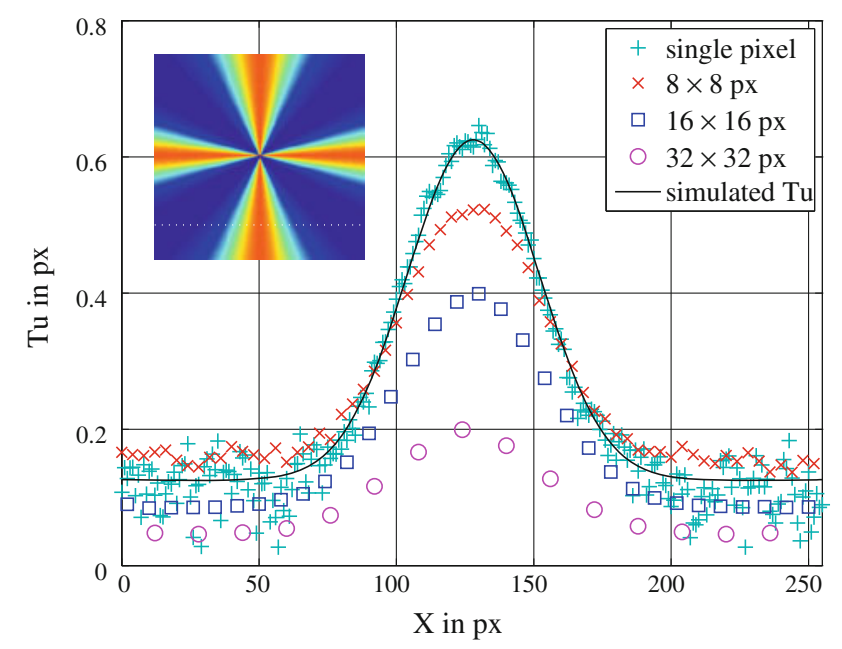

Fig. 13 Turbulence level for a cross-section at $y=50 \mathrm{px}$ of the data set from Fig. 12. The black solid line indicates the simulated turbulence level

smaller than the simulated values. This is due to the fact that the information is averaged over the window size.

Figures 13 and 14 illustrate how the correlation window size influences the magnitude of the estimated stresses. Furthermore, for the window size of $32 \mathrm{px} \times 32 \mathrm{px}$ and $16 \mathrm{px} \times 16 \mathrm{px}$, the absolute values are always too small, and for $8 \mathrm{px} \times 8 \mathrm{px}$ windows, the number of spurious vectors increases significantly, which introduces artificial stresses. The maximum deviation between simulated turbulence level and estimation using ensemble correlation is only $0.10 \mathrm{px}$ for the cross-section at $y=50 \mathrm{px}$, as shown in Fig. 13, whereas it is $0.32 \mathrm{px}$ in the case of $16 \mathrm{px} \times 16 \mathrm{px}$ window correlation. For the Reynolds shear stress, the maximum deviations in Fig. 14 are px and 


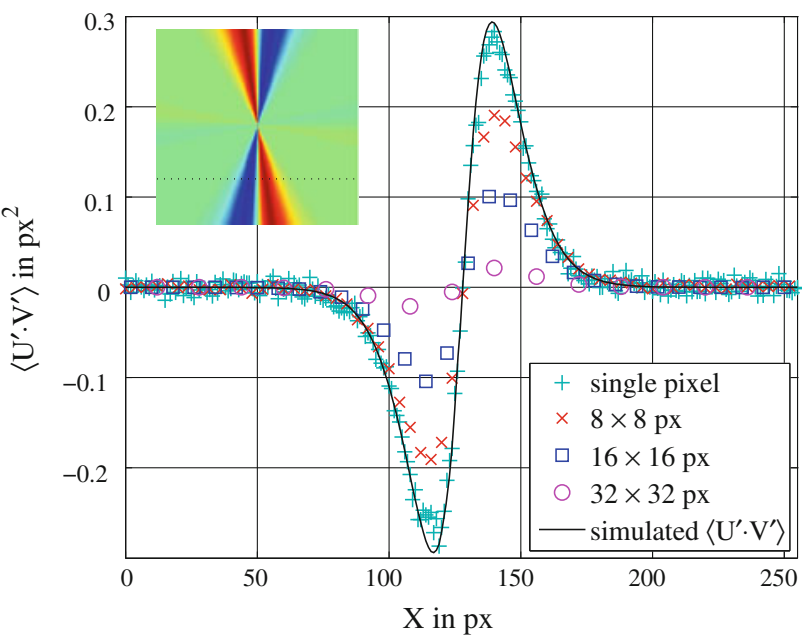

Fig. 14 Reynolds shear stress for a cross-section at $y=50$ px of the data set from Fig. 12. The black solid line indicates the simulated turbulence level

$0.25 \mathrm{px}$ for the ensemble correlation and the $16 \mathrm{px} \times$ 16 px window correlation, respectively.

The evaluated synthetic test case shows that window correlation-based PIV evaluation cannot resolve small turbulent structures; only turbulent structures larger than the interrogation window size contribute to the estimated stresses.

However, the ensemble correlation-based method is, in principle, capable to detect any small turbulent structures. It does not average velocity fluctuations over a certain area, not even over the single-pixel, and all fluctuations are stored within the correlation peak. Also, turbulent structures smaller than one pixel contribute to the broadening of the correlation peak even though the computed stresses correspond to the mean values over the area of one pixel.

\subsection{Compensation of velocity gradient effects}

In Sect. 2.3 (Fig. 1), the deformation of the correlation peak due to a gradient in the velocity field was demonstrated. This effect is quantitatively analyzed in this section, for which PIV images with homogeneous stresses were generated. The parameters $P_{X}, P_{Y}$, and $\alpha$ of the PDF were chosen to be $2,0.5 \mathrm{px}$, and $0^{\circ}$, respectively. The velocity gradient $\partial \Delta X / \partial Y$ varied from -1 to $+1 \mathrm{px} / \mathrm{px}$.

Figures 15, 16, and 17 show the computed normal stresses and shear stresses. Three different particle image diameters were tested. The figures show a strong bias error that depends on the particle image size and the velocity gradient. Only the normal stress in the $Y$-direction is not effected by the velocity gradient.

The strong bias error is caused by the deformation of the correlation peaks in the case of velocity gradients. Figure 18 shows the correlation peaks for the first, last, and

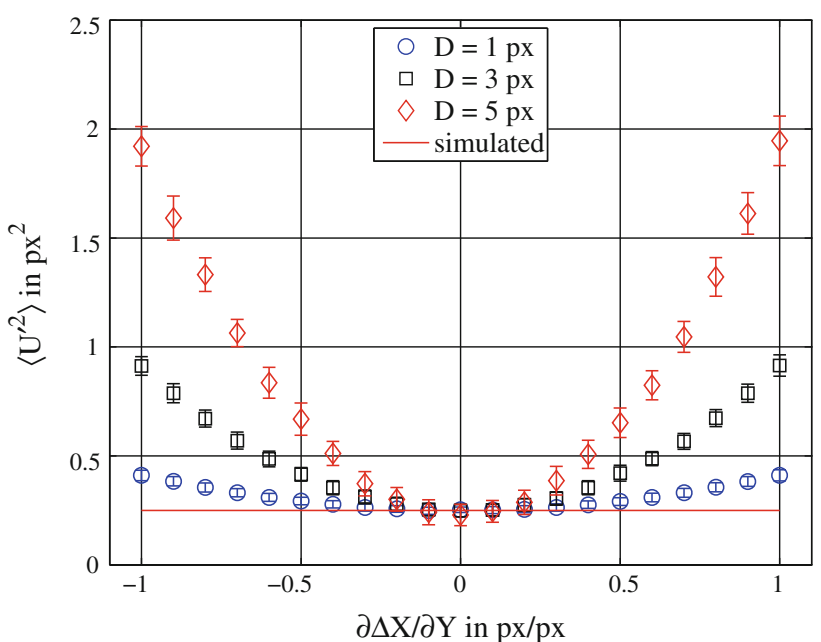

Fig. 15 Reynolds normal stress in the $X$-direction under the influence of the velocity gradient $\partial \Delta X / \partial Y$

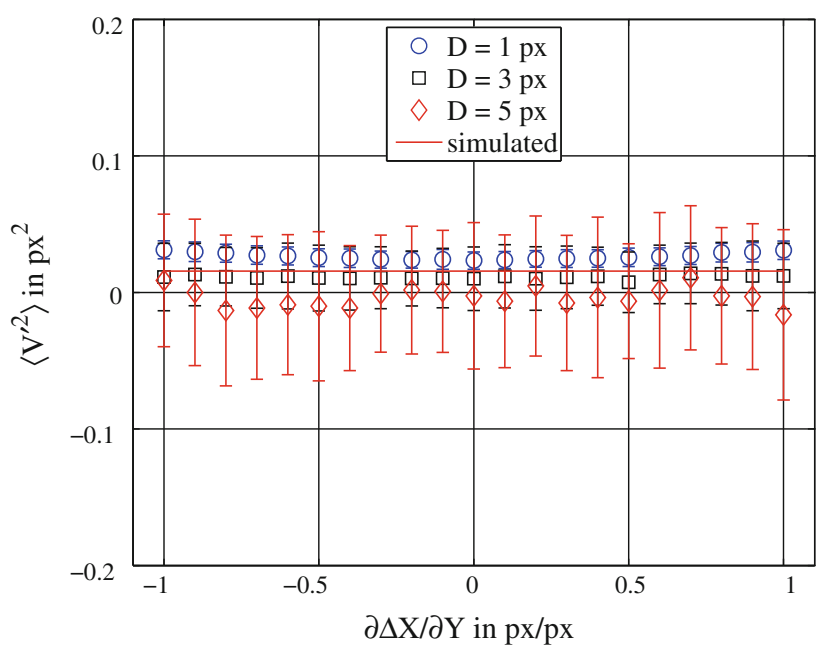

Fig. 16 Reynolds normal stress in the $Y$-direction under the influence of the velocity gradient $\partial \Delta X / \partial Y$

middle points of the data set from Figs. 15, 16, and 17 for $D=5$ px. These peaks are computed by using Eq. 5 and are in qualitative agreement with the analytical functions in Fig. 1. The correlation peaks are stretched in the direction of the flow and compressed perpendicular to it. Additionally, a positive velocity gradient $\partial \Delta X / \partial Y$ rotates the correlation peak in the counter clockwise direction and vice versa.

Figures 15, 17, and 18 clearly show that the simple relations from $13 \mathrm{a}$ and $\mathrm{b}$ are not sufficient if strong velocity gradients are present

A detailed inspection of Eq. 17 allows for a correction of the Reynolds stresses with respect to the velocity gradient $\partial \Delta X / \partial Y$ : 


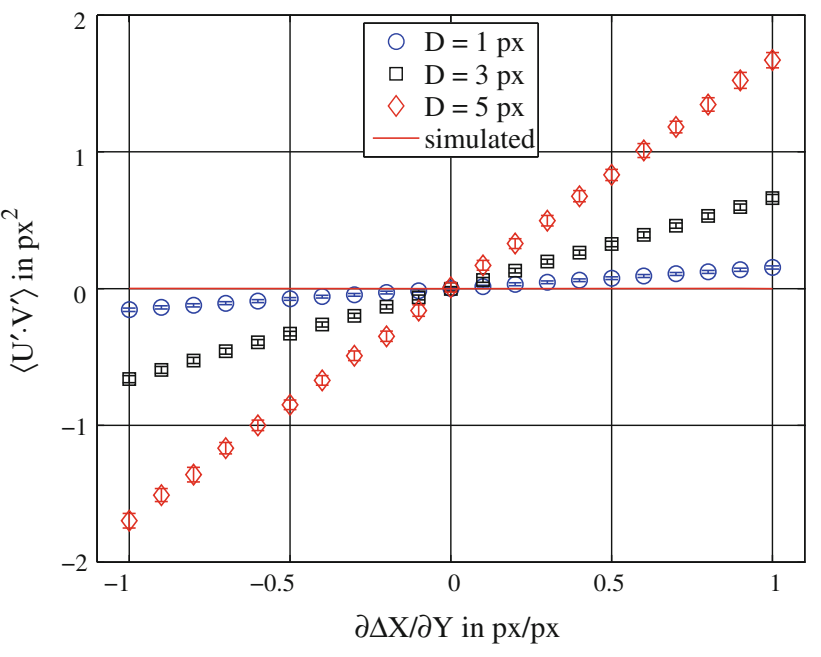

Fig. 17 Reynolds shear stress under the influence of the velocity gradient $\partial \Delta X / \partial Y$
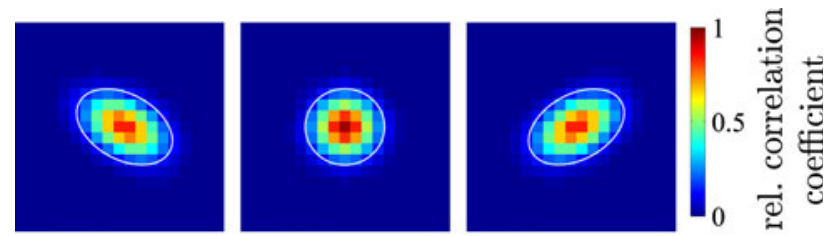

$$
\partial \Delta X / \partial Y=[-1,0,1]
$$

Fig. 18 Computed correlation peak for each 20,000 synthetic PIV image pairs with different velocity gradients $\partial \Delta X / \partial Y$. A particle image diameter of $D=5 \mathrm{px}$ was applied. The parameters $P_{X}, P_{Y}$, and $\alpha$ of the PDF were $2,0.5 \mathrm{px}$, and $0^{\circ}$, respectively. The solid lines indicate the size and orientation of the fitted Gaussian peak

$$
\begin{aligned}
& \left\langle U^{\prime 2}\right\rangle_{\text {corrected }}=\left\langle U^{\prime 2}\right\rangle_{\text {gradient }}-\frac{D^{2}}{16} \cdot\left(\frac{\partial \Delta X}{\partial Y}\right)^{2}, \\
& \left\langle V^{\prime 2}\right\rangle_{\text {corrected }}=\left\langle V^{\prime 2}\right\rangle_{\text {gradient }}-\frac{D^{2}}{16} \cdot\left(\frac{\partial \Delta Y}{\partial X}\right)^{2}
\end{aligned}
$$

and

$$
\left\langle U^{\prime} \cdot V^{\prime}\right\rangle_{\text {corrected }}=\left\langle U^{\prime} \cdot V^{\prime}\right\rangle_{\text {gradient }}-\frac{D^{2}}{16} \cdot\left(\frac{\partial \Delta X}{\partial Y}+\frac{\partial \Delta Y}{\partial X}\right) .
$$

For the derivation of Eqs. 24, 25, and 26, the analytical correlation peak from Eq. 17 was compared to a Gaussian peak with elliptical cross-section like the one used in Eq. 11. In this way, it was possible to identify the change of the parameters $C_{X}, C_{Y}$, and $\alpha$ with respect to the velocity gradient $\partial \Delta X / \partial Y$ and hence to correct the Reynolds stresses. Additionally, the influence of the velocity gradient $\partial \Delta Y / \partial X$ was investigated: both gradient effects can be corrected independently.

In order to verify Eqs. 24-26, 20,000 synthetic PIV images with a size of $256 \mathrm{px} \times 256 \mathrm{px}$ and varying velocity gradients were generated and evaluated. The particle image diameter was $D=3 \mathrm{px}$, and the parameters $P_{X}, P_{Y}$, and $\alpha$ were $2,2 \mathrm{px}$, and $0^{\circ}$, respectively. Isotropic Reynolds stresses were applied; thus, the shear stress should be zero.

Figure 19 shows the distribution of the horizontal velocity, the Reynolds shear stress influenced by the velocity gradient $\left\langle U^{\prime} \cdot V^{\prime}\right\rangle_{\text {gradient }}$ and the corrected shear stress $\left\langle U^{\prime} \cdot V^{\prime}\right\rangle_{\text {corrected }}$ computed with Eq. 26. In the right column of Fig. 19, the projection of the data points on the $Y$-axis is shown together with the simulated values (red solid line). A sinusoidal velocity profile with increasing amplitude from top to bottom was generated (Fig. 19a). Thus, the velocity gradient also increased and the Reynolds shear stress seemed to rise with it (Fig. 19b).

Note, however, that the estimation of the mean velocity is not affected. Thus, it is possible to determine the velocity gradient from the mean velocity field and to correct the
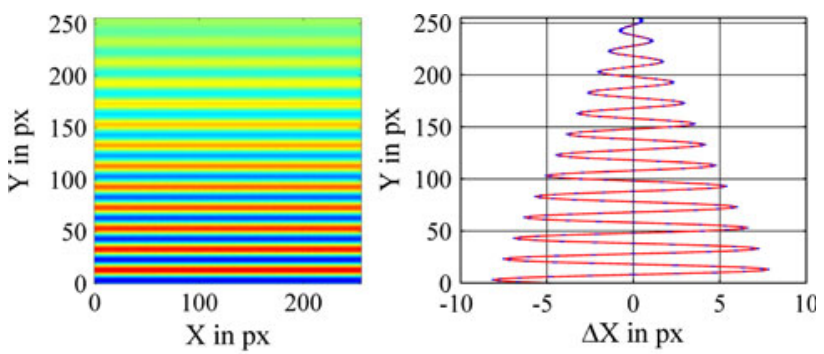

(a)
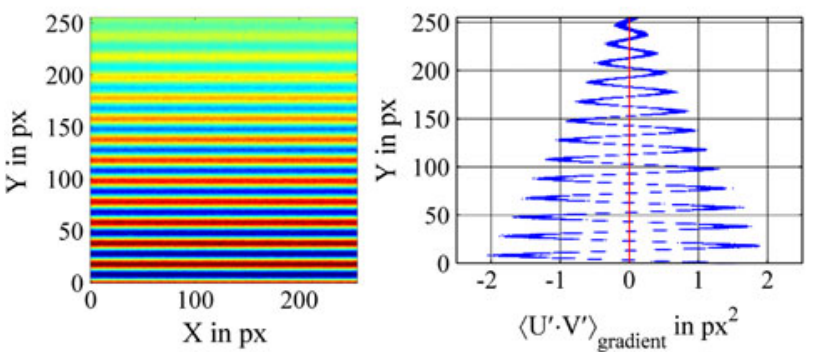

(b)
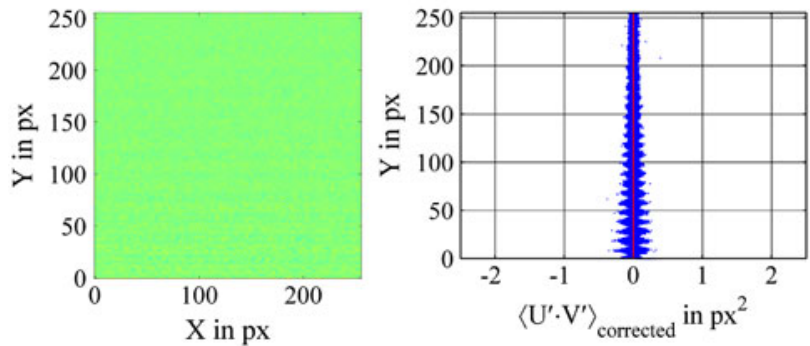

(c)

Fig. 19 Influence of the velocity gradient $\partial \Delta X / \partial Y$ on the Reynolds shear stress for a synthetic shear flow with varying gradient. 20,000 synthetic PIV images with isotropic stresses were analyzed. a horizontal velocity, b uncorrected Reynolds shear stress, c corrected Reynolds shear stress 
Reynolds stresses. A correction, using Eq. 26, of the shear stress is shown in Fig. 19c. The remaining standard deviation of the corrected shear stress $\left\langle U^{\prime} \cdot V^{\prime}\right\rangle_{\text {corrected }}$ is only $0.045 \mathrm{px}^{2}$ (for the uncorrected stress it was $0.7 \mathrm{px}^{2}$ ). An other test case with both gradients $\partial \Delta X / \partial Y$ and $\partial \Delta Y / \partial X$ (not presented in this work) confirmed that these can be compensated independently.

In conclusion, it can be stated that the estimation of the Reynolds stresses is strongly influenced by the velocity gradients $\partial \Delta X / \partial Y$ and $\partial \Delta Y / \partial X$. It was found that the computed values can be categorized between real and artificial stresses. The latter depend on the particle image diameter squared, for all in-plane Reynolds stress components. The analytical results are in good agreement with the computed values from the synthetic PIV recordings.

It should be emphasized again that only the first derivative of $u$ with respect to $y$ was considered for the derivation of the correlation function (see Eq. 16). However, a higher order expansion might lead to even better results.

\section{Analysis of real PIV images}

So far, only synthetic PIV images were analyzed. This section presents computed Reynolds stresses for real PIV recordings using the method discussed previously. Section 4.1 focuses on a round jet flow in water, and Sect. 4.2 presents results of a space launcher model's wake in air at a transonic Mach number. Only the shear stresses are presented in this report, since these are usually of major interest as long as no flow separation is studied in detail. Nevertheless, the normal stresses require almost no additional computing time and can easily be extracted for the complete description.

\subsection{Water jet flow}

The first experimental example analyzes a round jet flow in water at a Reynolds number of $R e_{d} \approx 26,000$ based on the nozzle diameter which was $\emptyset d=2.4 \mathrm{~mm}$. The pipe had an inner diameter of approximately $10 \mathrm{~mm}$ and was connected to a diaphragm pump. The setup is illustrated in Fig. 20.

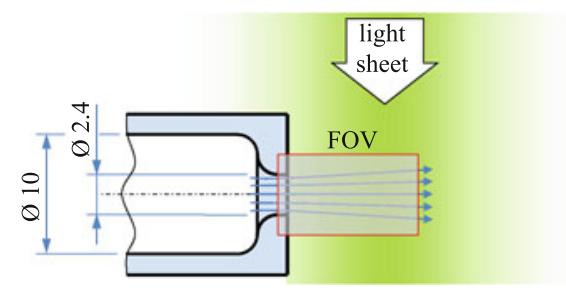

Fig. 20 Sketch of the axis-symmetric pipe exit for the jet flow analysis. The laser light sheet and the field of view (FOV) for PIV are illustrated. Numerical values are given in $\mathrm{mm}$
The near field of the jet was selected, as the resolution of the strong gradient at the outlet of the nozzle is very difficult.

A high repetition rate CMOS camera (Phantom V12 by Vision Research Inc.) with a Zeiss Makro-PlanarT* ${ }^{*} 2 /$ 100 objective lens plus a $2 \times$ teleconverter (Kenko $2 \times$ Pro 300) was used to observe the jet. The working distance was approximately $0.5 \mathrm{~m}$, which led to an optical magnification of $M=0.73$ and a scaling factor of $27.5 \mu \mathrm{m} /$ px. Twenty-five thousand single-exposed PIV image pairs with a size of $384 \mathrm{px} \times 256 \mathrm{px}$ were captured at a frequency of $250 \mathrm{~Hz}$, while the interframing time was set to $\Delta t=20 \mu \mathrm{s}$. The exposure time of the camera was set to $1.8 \mu$ s. A continuous-wave laser (Millennia by SpectraPhysics) with $10 \mathrm{~W}$ optical power and a wave length of $532 \mathrm{~nm}$ was used to form a light sheet of approximately $300 \mu \mathrm{m}$ in thickness. Hollow class spheres with a diameter of $10 \mu \mathrm{m}$ were used as tracer particle (by Dantec Dynamics).

The analysis of the PIV recordings revealed that the particle image diameter on the image plane was around $D \approx 1.9 \mathrm{px}$, and the particle density was such that approximately $1 \%$ of the images was illuminated, this corresponds to about 1.3 particle images in an area of

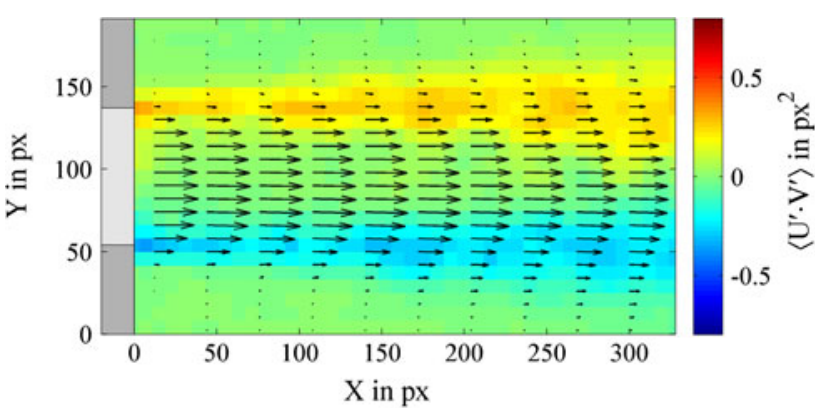

(a)

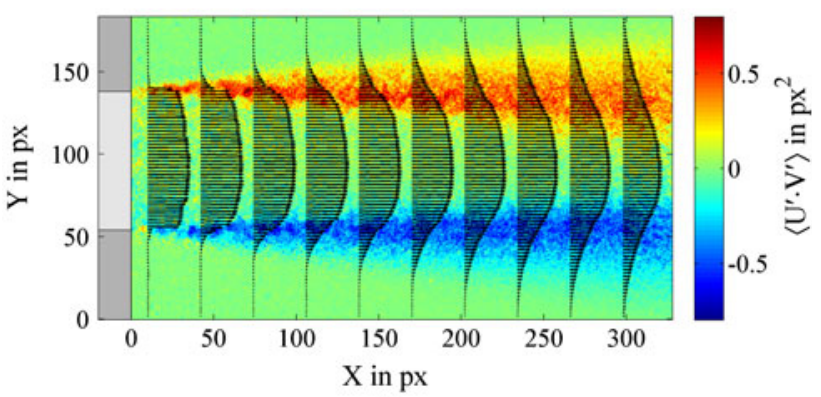

(b)

Fig. 21 Reynolds shear stress distribution and velocity vectors for a round water jet at $R e=22,000$ computed with single-pixel resolution (bottom) and for $16 \mathrm{px} \times 16 \mathrm{px}$ interrogation windows (top) from 25,000 PIV recordings. a $16 \mathrm{px} \times 16 \mathrm{px}$ window correlation: every 4th vector in $X$-direction and each vector in $Y$-direction is shown. b Ensemble correlation: every 32 nd vector in $X$-direction and every 2nd vector in $Y$-direction is shown 
$16 \mathrm{px} \times 16 \mathrm{px}$. The maximum velocity of the jet was determined to be $9.77 \mathrm{~m} / \mathrm{s}$ corresponding to a shift vector of $7.1 \mathrm{px}$.

Figure 21 shows a comparison of the Reynolds shear stress distribution directly behind the nozzle, between a window correlation method using a standard software (Davis by LaVision $\mathrm{GmbH}$ ) and the developed single-pixel ensemble correlation approach.

A multi-pass algorithm with decreasing window size (from $32 \mathrm{px} \times 32 \mathrm{px}$ to $16 \mathrm{px} \times 16 \mathrm{px}$ with $50 \%$ overlap) and a Gaussian window weighting was applied for the window correlation method. Thus, a total of 252 independent vectors could be computed for 25,000 vector fields leading to 252 independent data points for the Reynolds shear stress distribution, computed from this ensemble of vector fields (Fig. 21a).

On the other hand, for the single-pixel approach, 59,400 correlation peaks were computed leading to 59,400 independent Reynolds shear stress data points (Fig. 21b). A locally active filter was used to replace single outliers by the median value of the surroundings.

The comparison between window correlation and ensemble correlation shows a similar distribution: a shear layer with positive shear stress in the upper part and one with negative shear stress in the lower part. Both shear layers grow in thickness with propagation, while the broadening is proportional to the distance from the nozzle. Although, this measurements were not performed in the self-preserving region, this results are in agreement with the analysis on the self-preserving region of a round jet made by of Wygnanski and Fiedler (1969).

Regarding the comparison in Fig. 21, a significantly better spatial resolution was achieved with the developed single-pixel method. Furthermore, it is evident that the absolute values of the shear stresses are considerably higher in the case of single-pixel evaluation. Referring to the example of synthetic PIV images from Fig. 12, these values are more reliable than those obtained from window correlation. The single-pixel results are neither spatially low-pass filtered by the correlation window size, nor do they suffer from error propagation during the calculation of Reynolds stresses from individual vector fields.

Additionally, the velocity vectors in Fig. 21 clearly show that only the single-pixel approach is capable of resolving the very high velocity gradients right behind the nozzle exit. The window correlation method only computes mean values averaged over the window size.

A cross-section through the shear stress distribution at $X=150 \mathrm{px}$ is shown in Fig. 22 for the single-pixel approach and for window correlation-based evaluation using three different window sizes. With window correlation, only the stresses generated by turbulent structures larger than the window size are detected, because each

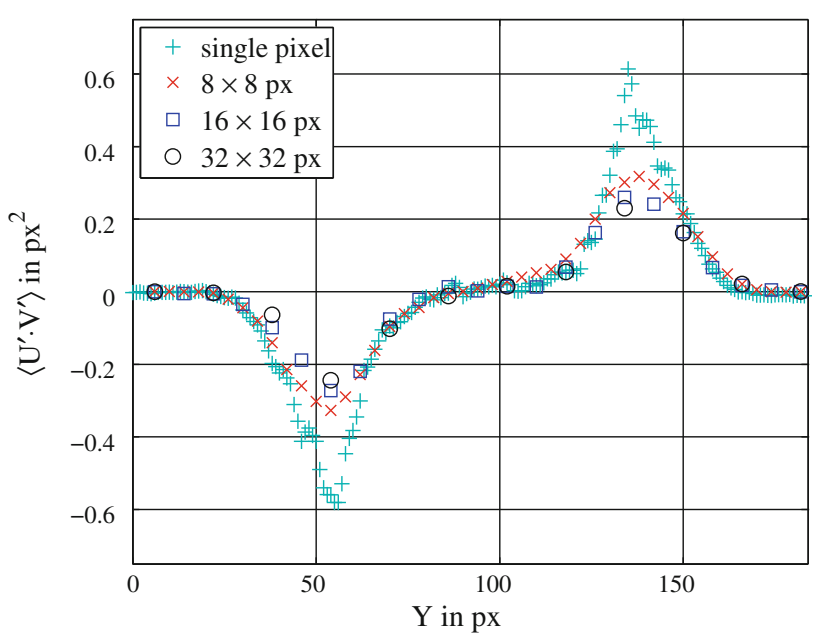

Fig. 22 Reynolds shear stress for a cross-section of the jet flow from Fig. 21 at $x=150 \mathrm{px}$

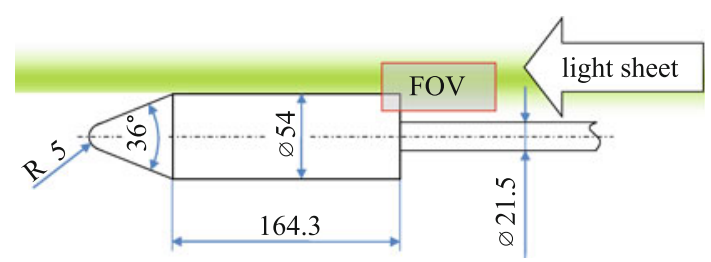

Fig. 23 Axis-symmetric space launcher model with rear sting. The laser light sheet and the field of view (FOV) for PIV are illustrated. Numerical values are given in $\mathrm{mm}$

vector represents only the mean velocity of the interrogation window. That is why the absolute values of the stresses are underestimated.

\subsection{Space launcher model wake flow}

The second experimental example analyzes the wake flow of a blunt axis-symmetric space launcher model as shown in Fig. 23. The configuration consists of a $36^{\circ}$ cone with a spherical nose of $R=5 \mathrm{~mm}$, a cylindrical part of $164.3 \mathrm{~mm}$ in length and a diameter of $\varnothing D=54 \mathrm{~mm}$. A rear sting $(\varnothing d=21.5 \mathrm{~mm})$ in the base of the cylinder was used for mounting the model in the test section of the wind tunnel. Detailed information about the measurement setup and the image preprocessing, as well as further results, can be found in Bitter et al. (2010).

The analyzed data set was acquired at a Mach number of $\mathrm{Ma}=0.7$ and a Reynolds number of $R e_{D}=10^{6}$ (related to the diameter $\varnothing D=54 \mathrm{~mm}$ ). The evaluation is based on a total number of 5,000 PIV recordings. The particle image density was such that approximately $3.0 \%$ of the image was illuminated and the particle image diameter was 1.7$2.2 \mathrm{px}$. Hence, about $2-5$ particle images were within an area of $16 \mathrm{px} \times 16 \mathrm{px}$. 
Figure $24 \mathrm{a}$ and $\mathrm{b}$ show the comparison between evaluation with the standard software (Davis by LaVision $\mathrm{GmbH})$ and the developed single-pixel approach for the velocity field and the Reynolds shear stress distribution. In Figure 24 , only every 4 th velocity vectors is displayed. The interrogation windows had a size of $16 \mathrm{px} \times 16 \mathrm{px}$ and were overlapped by $50 \%$ in each direction. Hence, a total of 160 independent data points was achieved. Due to the low seeding density, the interrogation window size can not be reduced any further.

For the vector field in Fig. 24b, every 32nd vector in the $X$-direction and every 2 nd in the $Y$-direction are displayed. There is a strong out-of-plane component due to the threedimensional behavior of the flow. Thus, a stereoscopic analysis with single-pixel resolution would be needed, as outlined in Scholz and Kähler (2006). To compensate for the out-of-plane losses, the correlation peaks were averaged over $6 \mathrm{px}^{2} \times 3 \mathrm{px}^{2}$ with a Gaussian weighting, meaning that the neighboring correlation peaks were multiplied with a number smaller 1 (depending on the distance) and added to the primary one prior applying the fit-function. Altogether 2,000 independent data points were computed.

The comparison in Fig. 24 shows increased spatial resolution for the velocity field and the shear stress distribution. The boundary layer along the cylindrical part of the launcher model was resolved down to $800 \mu \mathrm{m}$ with the standard PIV software and down to $200 \mu \mathrm{m}$ with the single-pixel approach. However, the viscous sublayer and the decay of the stream-wise velocity component to zero at the wall could not be resolved with the PIV setup used. Therefore, a long-range micro-PIV approach is required (Kähler et al. 2006).

Regarding the shear layer, the Reynolds stress distribution indicates a strong turbulent intensity. A broadening of the shear layer toward the reattachment point indicates unsteady fluctuations of the reattachment point and a strong momentum transfer between the outer free stream flow and the wake flow caused by vortex shedding at the base. The results reveal a generally good agreement with the standard PIV evaluation. Like before, the estimated stresses by using the single-pixel approach are significantly larger at the beginning of the shear layer. This is due to the spatial low-pass filtering of the window correlation method. Although, the experimental single-pixel results may suffer from unknown bias errors that were not included in the theory, they are more realistic than the window correlation results.

A cross-section through the shear stress distribution at $X=50$ px is shown in Fig. 25 (on the left side) for the single-pixel approach and for window correlation. Again, the single pixel approach results in much higher and more realistic values for the shear stress than the window correlation method. This is due to the fact that window
Fig. 24 Velocity field and Reynolds shear stress distribution of the wake flow of a space launcher model. a $16 \mathrm{px} \times 16 \mathrm{px}$ window correlation: every 4 th vector in $X$-direction and each vector in $Y$-direction is shown.

b Ensemble correlation: every 32 nd vector in $X$-direction and every 2 nd vector in $Y$-direction is shown. The correlation peaks were averaged over $6 \mathrm{px} \times 3 \mathrm{px}$

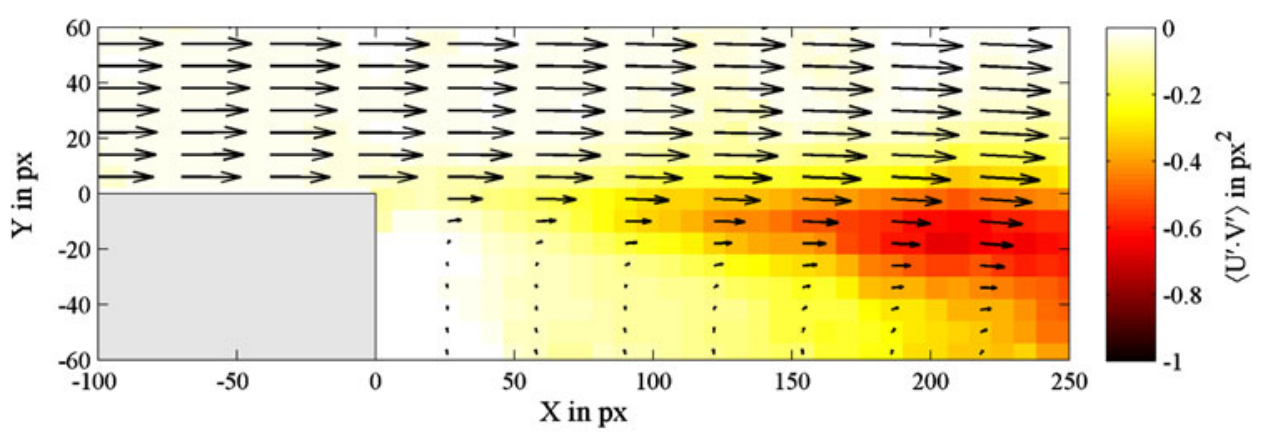

(a)

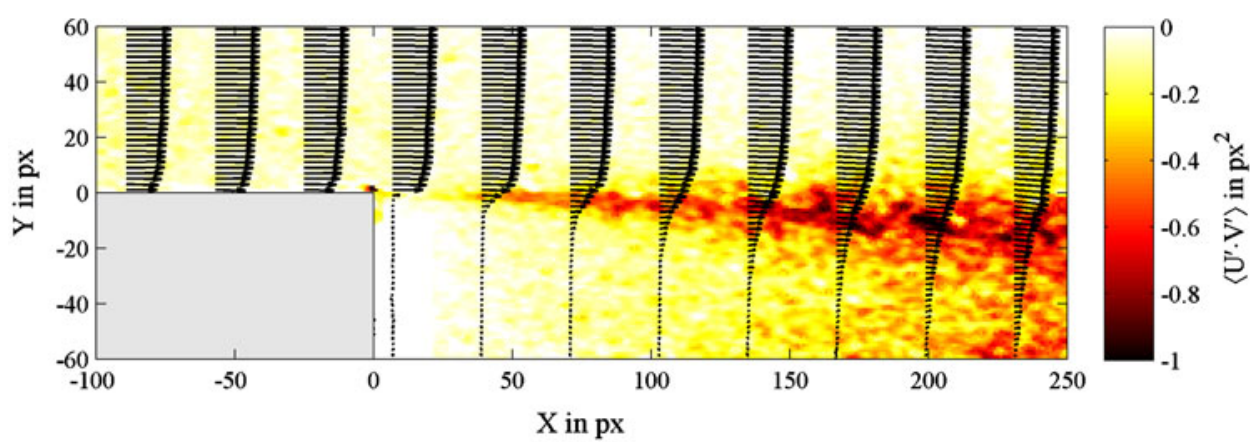

(b) 

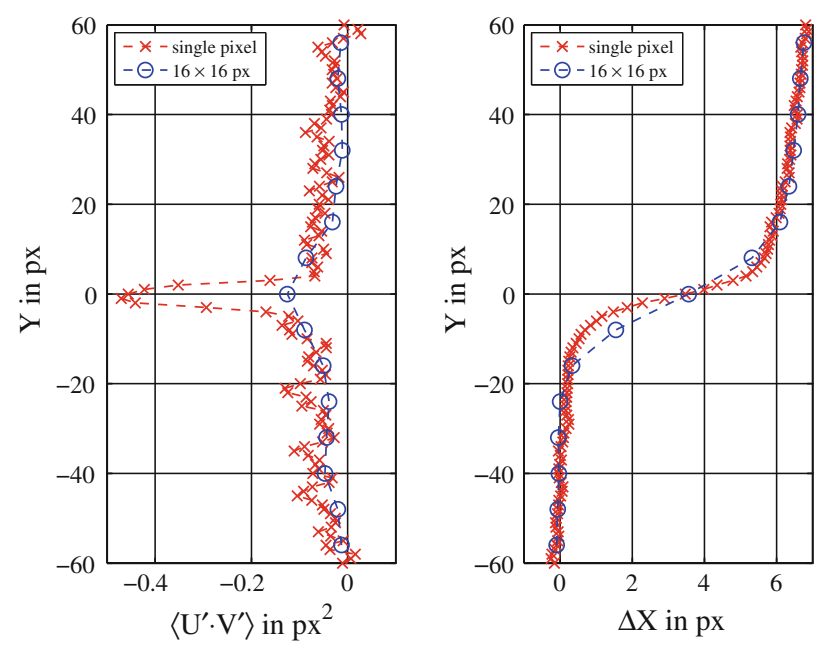

Fig. 25 Reynolds shear stress and horizontal velocity component for a cross-section of the shear layer from Fig. 24 at $x=50 \mathrm{px}$

correlation-based PIV evaluation methods underestimate the absolute values of the stresses, as demonstrated in Sect. 3.4 for synthetic PIV data.

Additionally, the right hand plot of Fig. 25 shows the profile of the horizontal velocity component at $X=50$ px. The window correlation method is not capable of precisely determining the velocity in the case of strong curvature in the profile. There is a maximum difference of $0.8 \mathrm{px}$ for the shift vector at $Y=-8 \mathrm{px}$. As a consequence, the velocity gradient at $Y=0 \mathrm{px}$ is overestimated.

\section{Conclusion}

The presented work illustrates that Reynolds normal and shear stresses can be estimated in general from the shape of the correlation function. This allows for computing statistical values with single-pixel resolution.

The developed method is suitable for PIV recordings with particle image diameters $D \geq 1$ px (see Figs. 4, 5), whereas the optimum value is between 1.5 and $5 \mathrm{px}$.

The number of images has a strong impact on the accuracy of the computed stresses and the mean velocity (see Figs. 7, 8, and 9). An insufficient number of PIV images (or low seeding density) can be compensated by averaging the correlation peaks over several pixels.

Velocity gradients in the observed flow stretch the correlation peaks and rotate them. This causes strong bias errors for the computed Reynolds stresses, which must be corrected by using Eqs. 24-26.

The application of the evaluation method on two experimental data sets in Sect. 4 demonstrated the suitability of this approach for the analysis of real PIV recordings. The comparison between the developed single- pixel approach and a standard evaluation using interrogation windows illustrates the increased spatial resolution and enhanced measurement precision (see Figs. 21, 24). It was found that the estimated Reynolds stresses are significantly larger compared to results from standard PIV evaluations that only compute mean values averaged over the interrogation window size.

In summary, for a PIV data set of several thousand image pairs, all Reynolds stresses in a 2-D regime can be computed with single-pixel resolution, with an error of only a few percent points. This is of great importance for the analysis of small scale flow phenomena appearing, for instance, at large Reynolds and Mach numbers. In principle, the developed method can also be used to analyze correlation peaks from sum-of-correlation procedures, computed with conventional available software. However, in this case, the received information is averaged over the interrogation window size.

The present calculations are based on a Gaussian probability density function. For instationary flows with complex behavior, the probability density function might have to be generalized. A sum of two or more Gaussian functions like the one defined in Eq. 9 should solve a variety of problems.

Acknowledgments Financial support from the German Research Foundation (Deutsche Forschungsgemeinschaft-DFG) in the framework of the Sonderforschungsbereich Transregio 40 "Technological foundations for the design of thermally and mechanically highly loaded components of future space transportation systems" is gratefully acknowledged by the authors.

Open Access This article is distributed under the terms of the Creative Commons Attribution Noncommercial License which permits any noncommercial use, distribution, and reproduction in any medium, provided the original author(s) and source are credited.

\section{References}

Arnold W, Hinsch KD, Mach D (1986) Turbulence level measurement by speckle velocimetry. Appl Opt 25:330-331

Billy F, David L, Pineau G (2004) Single pixel resolution correlation applied to unsteady flow measurements. Meas Sci Technol 15:1039-1045

Bitter M, Hain R, Müller J, Kähler CJ (2009) Characterization of boundary- and wake-flows up to $\mathrm{Ma}=2.5 \mathrm{using}$ high-speed long-range micro-PIV. In: 8th international symposium on particle image velocimetry-PIV09, Melbourne

Bitter M, Scharnowski S, Hain R, Kähler CJ (2010) High repetitionrate PIV investigations on a generic rocket model in sub- and supersonic flows. Exp Fluids 50:1019-1030. doi:10.1007/ s00348-010-0988-8

Born M, Wolf E (2000) Principles of optics. Cambridge University Press, Cambridge

Chamarthy P, Garimella SV, Wereley ST (2009) Non-intrusive temperature measurement using microscale visualization techniques. Exp Fluids 47:159-170 
Hohreiter V, Wereley ST, Olsen MG, Chung JN (2002) Crosscorrelation analysis for temperature measurement. Meas Sci Technol 13:1072-1078

Kähler CJ (1997) Ortsaufgelöste Geschwindigkeitsmessungen in einer turbulenten Grenzschicht. DLR-FB 97-32

Kähler CJ, Scholz U (2006) Transonic jet analysis using long-distance micro PIV. In: 12th international symposium on flow visualization, Göttingen, Germany

Kähler CJ, Scholz U, Ortmanns J (2006) Wall-shear-stress and near wall turbulence measurements up to single pixel resolution by means of long-distance micro-PIV. Exp Fluids 41:327-341

Keane RD, Adrian RJ (1990) Optimization of particle image velocimeters. Part I: double pulsed systems. Meas Sci Technol $1: 1202-1215$
Raffel M, Willert CE, Wereley ST, Kompenhans J (2007) Particle image velocimetry. Springer-Verlag, Berlin

Scholz U, Kähler CJ (2006) Dynamics of flow structures on heaving and pitching airfoils. In: 13th international symposium on applications of laser techniques to fluid mechanics, Lisbon

Westerweel J, Geelhoed PF, Lindken R (2004) Single-pixel resolution ensemble correlation for micro-PIV applications. Exp Fluids 37:375-384

Wygnanski I, Fiedler H (1969) Some measurements in the selfpreserving jet. J Fluid Mech 38:577-612 